Homotopy Decompositions and K-theory of Bott Towers

Civan, Yusuf and Ray, Nigel

2004

MIMS EPrint: 2005.34

Manchester Institute for Mathematical Sciences

School of Mathematics

The University of Manchester

\footnotetext{
Reports available from: http://eprints.maths.manchester.ac.uk/

And by contacting: The MIMS Secretary

School of Mathematics

The University of Manchester

Manchester, M13 9PL, UK
} 


\title{
HOMOTOPY DECOMPOSITIONS AND K-THEORY OF BOTT TOWERS
}

\author{
YUSUF CIVAN AND NIGEL RAY
}

\begin{abstract}
We describe Bott towers as sequences of toric manifolds $M^{k}$, and identify the omniorientations which correspond to their original construction as complex varieties. We show that the suspension of $M^{k}$ is homotopy equivalent to a wedge of Thom complexes, and display its complex $K$-theory as an algebra over the coefficient ring. We extend the results to $K O$-theory for several families of examples, and compute the effects of the realification homomorphism; these calculations breathe geometric life into Bahri and Bendersky's analysis of the Adams Spectral Sequence [2]. By way of application we consider the enumeration of stably complex structures on $M^{k}$, obtaining estimates for those which arise from omniorientations and those which are almost complex. We conclude with observations on the rôle of Bott towers in complex cobordism theory.
\end{abstract}

\section{INTRODUCTION}

In their 1950s study of loops on symmetric spaces, Bott and Samelson [4] introduced a remarkably rich and versatile family of smooth manifolds. Various special cases were treated in different contexts during the following three decades, until Grossberg and Karshon [14] offered a description as complex algebraic varieties in 1994. They referred to their constructions as Bott towers, and addressed issues of representation theory and symplectic geometry. Our purpose here is to offer the alternative viewpoint of algebraic topology, in the context of Davis and Januszkiewicz's toric manifolds [10]. We consider Bott towers $\left(M^{k}: k \leq n\right)$ of height $n$, and discuss homotopy decompositions of the suspensions $\Sigma M^{k}$; these provides further evidence that the spaces of complex geometry are often stably homotopy equivalent to wedges of Thom complexes, as we have argued elsewhere [13]. We investigate the real and complex $K$ theory of the $M^{k}$, casting geometric light on recent calculations of Bahri and Bendersky [2] which were originally conducted in the algebraic underworld of the Adams Spectral Sequence.

Given a commutative ring spectrum $E$, we denote the reduced and unreduced cohomology algebras of any space $X$ by $E^{*}(X)$ and $E^{*}\left(X_{+}\right)$respectively. So $E^{*}\left(S^{n}\right)$ is a free module over the coefficient ring $E_{*}$ on a single $n$-dimensional generator $s_{n}^{E}$, defined by the unit of $E$. In particular, we use this notation for the integral Eilenberg-Mac Lane spectrum $H$ and the complex $K$-theory spectrum $K$. Real $K$-theory requires the most detailed calculations, so we abbreviate $s_{n}^{K O}$ to $s_{n}$ whenever possible. We require multiplicative maps $f: E \rightarrow F$ of

Key words and phrases. Bott towers, K-theory, stably complex structures, Thom complexes, toric manifolds. 
ring spectra to preserve the units, so that $f\left(s_{n}^{E}\right)=s_{n}^{F}$ for all $n$; complexification $c: K O \rightarrow K$ is an important example. We adopt similar conventions for Thom classes $t^{E}$, which also play a major rôle. Given an $E$-orientable $n$-dimensional vector bundle $\gamma$, we insist that $t^{E}$ should lie in $E^{n}(T(\gamma))$, and restrict to $s_{n}^{E}$ on the fibre. Alternative choices of dimension are, of course, available for periodic spectra such as $K$ and $K O$, but we believe that our chosen convention leads to the least confusion.

With the single exception of $K O$, the spectra we use are complex oriented by an appropriate choice of first Chern class $v^{E}$ in $E^{2}\left(C P^{\infty}\right)$; by definition, $v^{E}$ restricts to $s_{2}^{E}$ on $C P^{1}$. We also insist that $E_{*}$ be concentrated in even degrees. So $v=v^{E}(\zeta(n))$ is the first Chern class of the Hopf line bundle $\zeta(n)$ over $C P^{n}$, and there is a canonical isomorphism

$$
E^{*}\left(C P_{+}^{n}\right) \cong E_{*}[[v]] /\left(v^{n+1}\right)
$$

for every $0 \leq n \leq \infty$. In order to emphasise that we are working over $C P^{n}$, we occasionally denote $v$ by $v(n)$; thus $v(1)$ and $s_{2}^{E}$ are interchangeable. In the cases $E=H$ and $K$, we write $v$ as $x$ and $u$ respectively.

Throughout our work, we express the coefficients of complex $K$-theory as the ring of Laurent series

$$
K_{*}=\mathbb{Z}\left[z, z^{-1}\right],
$$

where $z$ lies in $K_{2}$ and is represented by the virtual Hopf line bundle over $S^{2}$. Complex conjugation acts on $K_{*}$ by $\bar{z}=-z$.

For $K O$-theory, we denote the coefficient ring by

$$
K O_{*}=\mathbb{Z}[e, x, y] /\left(2 e, e^{3}, e x, 4 x^{2}-y\right),
$$

where $e, x$, and $y$ are represented by the real Hopf line bundle over $S^{1}$, the symplectic Hopf line bundle over $S^{4}$, and the canonical bundle over $S^{8}$ respectively, as described in [16], for example.

The contents of our sections are as follows.

We introduce Bott towers as toric manifolds in Section 2, relating the viewpoints of Davis and Januszkiewicz [10] and Grossberg and Karshon [14], and paying attention to the corresponding stably complex structures. In section 3 we interpret Bott towers as iterated sphere bundles, leading to an alternative derivation of their $E$-cohomology algebras, and splittings of their suspensions. We also consider associated properties of their stable tangent bundles, and introduce a cofiber sequence relating pairs of towers. Our calculations with $\mathrm{KO}$ theory begin in Section 4, where we focus on dimensions 2 and 4 and obtain complete descriptions of the $K O_{*}$-algebra structure in all cases. These results provide a springboard for our most comprehensive calculations, which occupy Section 5; we consider all dimensions, but specialise to two particular families of examples. Again, we obtain complete information about $K O_{*}$-algebra structures, but find that certain products are particularly complicated to describe explicitly. We also relate our results to the pioneering work of Bahri and Bendersky. In Section 6 we apply these calculations to the enumeration of certain collections of stably complex structures which arise from the toric viewpoint. Such structures are of key importance to understanding their rôle in complex cobordism theory. Section 7 acts as an appendix, to which readers should refer 
for notation, and for our treatment of the $E$-cohomology of certain sphere bundles $Y$ over complexes with cells in even dimensions. We record a homotopy decomposition of $\Sigma Y$, and give explicit details for the crucial cases $E=H$ and $K$.

The idea of studying Bott towers in this context first emerged during discussions with Victor Buchstaber, made possible by Aeroflot's abandonment of flights out of Manchester in the summer of 1996. The second author announced most of the results at the Conference on Algebraic Topology in Gdansk, Poland, during June 2001, where Taras Panov and his colleagues offered many helpful suggestions as we strolled the Baltic beaches. We apologise to them all for our protracted attempts to produce a final document, and give thanks to Adrian Dobson for identifying several errors in various intermediate versions.

We also acknowledge the influence of the referee, who instigated many improvements in the layout of the material.

\section{Toric Structures}

A Bott tower of height $n$ is a certain sequence of smooth oriented $2 k-$ dimensional manifolds $\left(M^{k}: k \leq n\right)$, determined by a list $\left(a_{1}, \ldots, a_{n-1}\right)$ of integral $(k-1)$-vectors

$$
a_{k-1}=(a(1, k), \ldots, a(k-1, k)) \text { for } 1<k \leq n .
$$

We begin by describing the $k$ th stage $M^{k}$ as a toric manifold $Q_{k}$, in the sense of Davis and Januszkiewicz [10]. We use the language of [6] to record additional salient properties, and discuss the relationship with Grossberg and Karshon's construction [14] of the $M^{k}$ as complex manifolds.

We write the $k$-dimensional torus as $T^{k}$ and denote a generic point $t$ by $\left(t_{1}, \ldots, t_{k}\right)$, where $t_{j}$ lies in the unit circle $T<\mathbb{C}$ for each $1 \leq j \leq k$. The $i$ th coordinate subcircle is the subgroup

$$
T_{i}=\left\{t: t_{j}=1 \text { for } j \neq i\right\} .
$$

The torus $T^{k}$ acts coordinatewise on $\mathbb{C}^{k}$, by multiplication; this is the standard representation, whose quotient is the nonnegative cone $\mathbb{R}_{\geqslant}^{k}$. Given a smooth, orientable $2 k$-dimensional manifold $L^{2 k}$, we consider actions of $T^{k}$ which are locally isomorphic to the standard representation, and whose quotient is homeomorphic to a simple convex polytope $P^{k}$. According to [10], the associated projection map $\pi: L^{2 k} \rightarrow P^{k}$ is a toric manifold.

Davis and Januszkiewicz show that every toric manifold may be represented by a characteristic function $\lambda$, which assigns a primitive vector in $\mathbb{Z}^{k}$ to each of the facets $F_{1}, \ldots, F_{m}$ of $P^{k}$. The vectors $\lambda\left(F_{i}\right)$ are uniquely defined up to sign; moreover, $\lambda\left(F_{i_{1}}\right), \ldots, \lambda\left(F_{i_{l}}\right)$ span an $l$-dimensional unimodular subspace of $\mathbb{Z}^{k}$ for every codimension- $l$ face $F=F_{i_{1}} \cap \cdots \cap F_{i_{l}}$ of $P^{k}$. Buchstaber and Ray [6] insist that the $\lambda\left(F_{i}\right)$ be directed, by choosing their signs. This is equivalent to providing an epimorphism $\ell: T^{m} \rightarrow T^{k}$, which they call a dicharacteristic. In these circumstances, $\lambda$ may be interpreted as a linear transformation $\mathbb{Z}^{m} \rightarrow \mathbb{Z}^{k}$, whose $k \times m$ matrix $\left(\lambda_{i, j}\right)$ describes the map of Lie algebras induced by $\ell$.

Davis and Januszkiewicz also provide a beautiful description of the integral cohomology ring $H^{*}\left(L^{2 k}\right)$, which is an analogue of the Danilov-Jurkiewicz 
Theorem for toric varieties. Their description involves the $(k-1)$-dimensional simplicial complex $K_{P}$, obtained by dualising the boundary of $P^{k}$. The StanleyReisner algebra of $K_{P}$ is the quotient $\mathbb{Z}\left[x_{1}, \ldots, x_{m}\right] / R$, where $R$ is the ideal generated by those monomials $x_{i_{1}} \cdots x_{i_{p}}$ which correspond to empty intersections $F_{i_{1}} \cap \cdots \cap F_{i_{p}}=\varnothing$.

Theorem 2.1 ([10], Theorem 4.14). The cohomology ring $H^{*}\left(L^{2 k}\right)$ is isomorphic to the quotient

$$
\mathbb{Z}\left[K_{P}\right] / \bar{J},
$$

where $\bar{J}$ is the ideal generated by the images of the elements

$$
\lambda_{j}=\lambda_{j, 1} x_{1}+\cdots+\lambda_{j, m} x_{m} \text { for } 1 \leq j \leq k .
$$

Every facet $F_{i}$ of $P^{k}$ lifts to a codimension-2 submanifold $X\left(F_{i}\right) \subset L^{2 k}$, with normal 2-plane bundle $\nu_{i}$. For any choice of dicharacteristic, the isotropy subgroup of $X\left(F_{i}\right)$ is the subcircle $\ell\left(T_{i}\right)<T^{k}$, and is therefore oriented by $\ell$; the associated tangent vector corresponds to $\lambda\left(F_{i}\right)$ in the Lie algebra of $T^{k}$. Since the isotropy subgroup acts on the normal fibres, it follows that $\nu_{i}$ is also oriented by $\ell$, for $1 \leq i \leq m$. The Pontryagin-Thom collapse maps $L^{2 k} \rightarrow T\left(\nu_{i}\right)$ define 2-plane facial bundles $\rho_{i}$ over $L^{2 k}$, and an orientation for $\nu_{i}$ is equivalent to an orientation for $\rho_{i}$. In other words, $\ell$ identifies $\rho_{i}$ as a complex line bundle for every $1 \leq i \leq m$, and reversing any of the constituent directions induces complex conjugation on the corresponding $\rho_{i}$.

An omniorientation for $L^{2 k}$ is given by an orientation and a choice of dicharacteristic. Following [6], the orientation equips the tangent bundle with an isomorphism

$$
\tau\left(L^{2 k}\right) \oplus \mathbb{R}^{2(m-k)} \cong \bigoplus_{i=1}^{m} \rho_{i},
$$

and the dicharacteristic invests the right-hand side with a complex structure. In other words, the omniorientation defines a canonical stably complex structure on $L^{2 k}$; this is preserved by the action of $T^{k}$, because the torus is connected. Changing the orientation of $L^{2 k}$ negates each structure.

In order to understand $Q_{k}$ from this viewpoint we study the standard action of $T^{2 k}$ on $\left(S^{3}\right)^{k}$, induced by embedding the latter in $\mathbb{C}^{2 k}$ as the subspace

$$
\left\{\left(y_{1}, z_{1}, \ldots, y_{k}, z_{k}\right): y_{j} \bar{y}_{j}+z_{j} \bar{z}_{j}=1 \text { for } 1 \leq j \leq k\right\} .
$$

When $k=1$ the quotient of this action is a curvilinear 1-simplex (or interval) $I=\left\{(r, s): r^{2}+s^{2}=1\right\}$ in $\mathbb{R}_{\geqslant}^{2}$, so for general $k$ it is a curvilinear cube $I^{k} \subset \mathbb{R}_{\geqslant}^{2 k}$.

Given any list $a=\left(a_{1}, \ldots, a_{k-1}\right)$, we write $T^{k}(a)<T^{2 k}$ for the $k$-dimensional subtorus consisting of elements

$$
\begin{aligned}
& \left\{\left(u_{1}, u_{1}, u_{2}, u_{1}^{-a(1,2)} u_{2}, \ldots, u_{j}, u_{1}^{-a(1, j)} \ldots u_{j-1}^{-a(j-1, j)} u_{j}, \ldots\right.\right. \\
& \left.\left.\ldots, u_{k}, u_{1}^{-a(1, k)} \ldots u_{k-1}^{-a(k-1, k)} u_{k}\right): u_{i} \in T \text { for } 1 \leq j \leq k\right\} .
\end{aligned}
$$

So $T^{k}(a)$ acts freely on $\left(S^{3}\right)^{k}$, and the quotient space $Q_{k}$ (or $Q^{k}(a)$, if the list requires emphasis) is a smooth $2 k$-dimensional manifold. Moreover, the $k$ torus $T^{2 k} / T^{k}(a)$ acts on $Q_{k}$, and has quotient $I^{k}$; we abbreviate $T^{2 k} / T^{k}(a)$ to 
$T_{a}^{k}$ whenever it acts on $Q_{k}$ in this fashion. Readers may check that this action is locally isomorphic to the standard representation, and that the projection map $Q_{k} \rightarrow I^{k}$ is a toric manifold. It is our toric model for $M^{k}$.

We follow [6] by writing the facets of $I^{k}$ as $C_{h}^{\varepsilon}$, where $1 \leq h \leq k$ and $\varepsilon$ is 0 or 1 . Here $C_{h}^{\varepsilon}$ is the $(k-1)$-cube $I^{h-1} \times(\varepsilon, 1-\varepsilon) \times I^{k-h}$ in $\mathbb{R}_{\geqslant}^{2 k}$, and $m=2 k$. We order the facets as

$$
C_{1}^{0}, C_{1}^{1}, \ldots, C_{h}^{0}, C_{h}^{1}, \ldots, C_{k}^{0}, C_{k}^{1}
$$

every intersection of the form $C_{h}^{0} \cap C_{h}^{1}$ is empty, whereas every other pair of facets shares a common face. For any choice of dicharacteristic $\ell: T^{2 k} \rightarrow T_{a}^{k}$, the isotropy subcircles of $X\left(C_{h}^{0}\right)$ and $X\left(C_{h}^{1}\right)$ are $\ell\left(T_{2 h-1}\right)$ and $\ell\left(T_{2 h}\right)$ respectively, for $1 \leq h \leq k$.

In order to describe a dicharacteristic explicitly, we pass to the corresponding homomorphisms of Lie algebras. By restriction, we obtain a short exact sequence

$$
\mathbb{Z}^{k}(a) \stackrel{\alpha}{\longrightarrow} \mathbb{Z}^{2 k} \stackrel{\lambda}{\longrightarrow} \mathbb{Z}_{a}^{k}
$$

of abelian groups. The monomorphism $\alpha$ is induced by (2.5), and its matrix is $2 k \times k$, with $j$ th column

$$
(0,0, \ldots, 0,0,1,1,0,-a(j, j+1), \ldots, 0,-a(j, k))
$$

for $1 \leq j \leq k$. We use the facets (2.6) as a basis for $\mathbb{Z}^{2 k}$, and note that the vectors $\left\{\lambda\left(C_{h}^{1}\right): 1 \leq h \leq k\right\}$ form a basis for $\mathbb{Z}_{a}^{k}$ because $\bigcap_{1 \leq h \leq k} C_{h}^{1}$ is a vertex of $I^{k}$. Finally, we choose our signs so as to ensure that the $k \times 2 k$ matrix $\left(\lambda_{i, j}\right)$ is given by

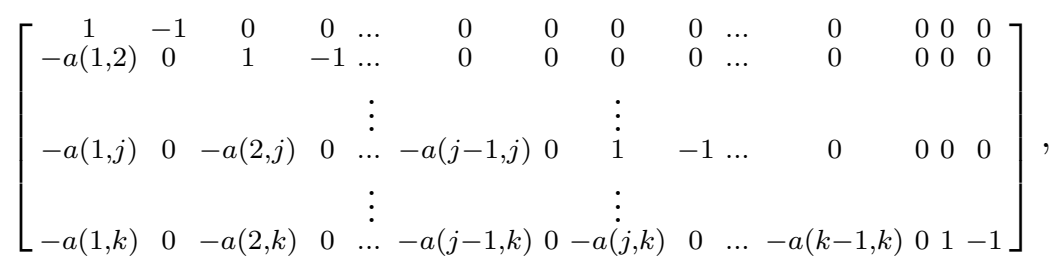

and the primitive vector associated to each facet of $I^{k}$ is read off from its columns. An orientation for $Q_{k}$ is given by combining our basis for $\mathbb{Z}_{a}^{k}$ with an orientation for $I^{k}$.

Remark 2.8. We could just as easily have begun with (2.7), and defined $Q_{k}$ up to equivariant diffeomorphism by Davis and Januszkiewicz's blowing down construction. Nevertheless, we prefer to start with $Q_{k}$; firstly because Bott and Samelson construct $M^{k}$ as a quotient of $\left(S^{3}\right)^{k}$, secondly because Grossberg and Karshon follow suit, and thirdly because Davis and Januszkiewicz themselves proceed in this order for classical cases such as $C P^{k}$.

Functions between cubes (or the corresponding products $\left.\left(S^{3}\right)^{k}\right)$ induce natural maps of the associated $Q_{k}$. For example, given $l<k$ we may consider the projection $I^{k} \rightarrow I^{k-l}$, defined by collapsing $C_{h}^{0}$ onto $C_{h}^{1}$ for any $l$ coordinates $h(1), \ldots, h(l)$; it admits left inverses $I^{k-l} \rightarrow I^{k}$, defined by identifying $I^{k-l}$ with $C_{h(1)}^{\epsilon(1)} \cap \cdots \cap C_{h(l)}^{\epsilon(l)}$ for any binary sequence $(\epsilon(q))$. These functions induce 
maps

$$
Q_{k}(a) \longrightarrow Q_{k-l}\left(a^{\prime}\right) \quad \text { and } \quad Q_{k-l}\left(a^{\prime}\right) \longrightarrow Q_{k}(a),
$$

where $a^{\prime}$ is obtained from $a$ by deleting all elements of the form $a(i, j)$ for which $i$ or $j=h(q)$ for some $1 \leq q \leq l$.

The simplicial polytope dual to $I^{k}$ is the $k$-dimensional crosspolytope [26], and the corresponding Stanley-Reisner algebra is given by

$$
\mathbb{Z}\left[K_{I^{k}}\right]=\mathbb{Z}\left[x_{1}, x_{1}^{\prime}, \ldots, x_{k}, x_{k}^{\prime}\right] /\left(x_{h} x_{h}^{\prime}=0: 1 \leq h \leq k\right),
$$

where $x_{h}$ and $x_{h}^{\prime}$ correspond to $C_{h}^{0}$ and $C_{h}^{1}$ respectively. We may now substitute the values for $\lambda_{i, j}$ of (2.7) into (2.2), and apply Theorem 2.1.

Corollary 2.10. The integral cohomology ring $H^{*}\left(Q_{k}\right)$ is isomorphic to the quotient $\mathbb{Z}\left[x_{1}, \ldots, x_{k}\right] / I_{k}$, where $I_{k}$ denotes the ideal

$$
\left(x_{j}\left(x_{j}-a(j-1, j) x_{j-1}-\cdots-a(1, j) x_{1}\right): 1 \leq j \leq k\right) .
$$

It is important to extend Corollary 2.10 to the complex oriented cohomology theories $E^{*}(-)$ of Section 1. As we shall see, this may be accomplished from the viewpoint of Section 3 by applying Lemma 7.2. Nevertheless, it also follows from a generalisation of Theorem 2.1, in which $\lambda_{j}$ is replaced by

$$
\lambda_{j}^{E}=F^{E}\left(\left[\lambda_{j, 1}\right]^{E}, \ldots,\left[\lambda_{j, k}\right]^{E}\right)
$$

for $1 \leq j \leq k$; here $F^{E}$ denotes the formal group law associated to $E$, and $[n]^{E}$ its $n$-series. The proof of this fact mimics that of Theorem 2.1.

Our construction of $Q_{k}$ leads immediately to the existence of canonical complex line bundles $\mu_{h}$, for $1 \leq h \leq k$. They are defined by

$$
\left(S^{3}\right)^{k} \times_{T^{k}(a)} \mathbb{C} \longrightarrow Q_{k},
$$

where $T^{k}(a)$ acts on $\mathbb{C}$ by $w \mapsto u_{h}^{-1} w$. Writing the facial bundles of $Q_{k}$ as $\rho_{h}^{0}$ and $\rho_{h}^{1}$, we deduce by the methods of [6] that (2.7) gives rise to isomorphisms $\rho_{h}^{0} \cong \bar{\mu}_{h}$ and $\rho_{h}^{1} \cong \bar{\mu}_{h} \otimes \mu\left(a_{h-1}\right)$ of real 2-plane bundles. So (2.3) converts the associated omniorientation into a stably complex structure

$$
\tau\left(Q_{k}\right) \oplus \mathbb{R}^{2 k} \cong \bigoplus_{h=1}^{k} \bar{\mu}_{h} \oplus\left(\bar{\mu}_{h} \otimes \mu\left(a_{h-1}\right)\right),
$$

where $\mu\left(a_{h-1}\right)$ stands for $\mu_{1}^{a(1, h)} \otimes \cdots \otimes \mu_{h-1}^{a(h-1, h)}$ in the notation of (7.1). Each of the other $2^{2 k}-1$ possible dicharacteristics defines an alternative structure, obtained by replacing the appropropriate facial bundles by their complex conjugates on the right-hand side of (2.12). These structures are important in complex cobordism theory, and we consider their enumeration in Section 6 .

Grossberg and Karshon [14] use a noncompact version of (2.5) to describe Bott towers as complex manifolds. Given a list $c=\left(c_{1}, \ldots, c_{k-1}\right)$ of integral vectors, they construct varieties $N_{k}$ as the quotient of $\left(\mathbb{C}^{2} \backslash 0\right)^{k}$ by a $k$-fold algebraic torus $\mathbb{C}_{\times}^{k}(c)$, under the action

$$
\begin{gathered}
\left(w_{1}, \ldots, w_{k}\right) \cdot\left(y_{1}, z_{1}, \ldots, y_{k}, z_{k}\right)= \\
\left(y_{1} w_{1}, z_{1} w_{1}, y_{2} w_{2}, w_{1}^{c(1,2)} z_{2} w_{2}, \ldots, y_{k} w_{k}, w_{1}^{c(1, k)} w_{2}^{c(2, k)} \ldots w_{k-1}^{c(k-1, k)} z_{k} w_{k}\right) .
\end{gathered}
$$


By mimicing the standard analysis for $C P^{k}$ [18], we deduce that the corresponding complex tangent bundle $\tau_{\mathbb{C}}\left(N_{k}\right)$ admits a canonical isomorphism

$$
\tau_{\mathbb{C}}\left(N_{k}\right) \oplus \mathbb{C}^{k} \cong\left(\mathbb{C}^{2} \backslash 0\right)^{k} \times_{\mathbb{C}_{\times}^{k}(c)} \mathbb{C}^{2 k},
$$

where $\mathbb{C}_{\times}^{k}(c)$ acts on $\mathbb{C}^{2 k}$ by extending (2.13).

As complex manifolds, $N_{k}$ coincides with $Q_{k}$, where the latter is determined by the list $a=-c$ (for which $a(i, j)=-c(i, j)$ for all $1 \leq i<j \leq k$ ). This observation is used in [9] to relate the quotient cube $I^{k}$ to the smooth fan determining $Q_{k}$. The right-hand side of (2.14) splits as a sum of line bundles, and yields the stably complex structure

$$
\tau_{\mathbb{C}}\left(Q_{k}\right) \oplus \mathbb{C}^{k} \cong \bigoplus_{h=1}^{k} \bar{\mu}_{h} \oplus\left(\bar{\mu}_{h} \otimes \mu\left(a_{h-1}\right)\right) .
$$

It follows that (2.12) and (2.15) agree, for the appropriate orientation of $Q_{k}$.

\section{Bott Towers}

In this section we introduce Bott towers as iterated sphere bundles $M^{k}$, and explain their relationship with the toric manifolds $Q_{k}$. We describe the cohomology rings $E^{*}\left(M^{k}\right)$ for any of the complex oriented ring spectra $E$, appealing repeatedly to the notation and background information of Section 7 . Our results extend earlier work [19] on bounded flag manifolds, and complement the more geometric approach of [9]. We obtain an elementary decomposition of the suspensions $\Sigma M^{k}$ into a wedge of Thom complexes, and consider two natural complex structures on their stable tangent bundles.

Given any integer $k \geq 1$, we assume that a $(k-1)$ th stage $M^{k-1}$ has been constructed as a smooth orientable $2(k-1)$-dimensional manifold with 2-generators $v_{j}^{E}$, and line bundles $\gamma_{j}$ such that $v^{E}\left(\gamma_{j}\right)=v_{j}^{E}$, for $1 \leq j \leq k-1$. As above, we write $\gamma\left(a_{k-1}\right)$ for the complex line bundle

$$
\gamma_{1}^{a(1, k)} \otimes \cdots \otimes \gamma_{k-1}^{a(k-1, k)}
$$

associated to a $(k-1)$-tuple $a_{k-1}=(a(1, k), \ldots, a(k-1, k))$ in $\mathbb{Z}^{k-1}$. Fixing $a_{k-1}$, we refer to $\gamma\left(a_{k-1}\right)$ as the kth bundle of the construction, and define $M^{k}$ to be the total space of the smooth 2 -sphere bundle of $\mathbb{R} \oplus \gamma\left(a_{k-1}\right)$; it is orientable by the complex structure on $\gamma\left(a_{k-1}\right)$ and choice of normal vector. By Lemma 7.2, we deduce that $M^{k}$ has 2-generators $v_{j}^{E}$ for $1 \leq j \leq k$, where $v_{k}^{E}$ is the pullback of the Thom class $t_{k}^{E} \in E^{2}\left(T\left(\gamma\left(a_{k-1}\right)\right)\right)$ along the collapse map $q_{k}$. Moreover, $t_{k}^{E}$ is the first Chern class of a canonical line bundle $\lambda_{k-1}$ over $T\left(\gamma\left(a_{k-1}\right)\right)$, so $v_{k}^{E}=v^{E}\left(\gamma_{k}\right)$, where $\gamma_{k}$ is defined as $q_{k}^{*} \lambda_{k-1}$. Henceforth, we abbreviate $T\left(\gamma\left(a_{j}\right)\right)$ to $T\left(a_{j}\right)$ for each $1 \leq j \leq n$.

In order to get off the ground, it is convenient to write the one-point space as $M^{0}$, so that the first bundle is trivial and $x_{0}=0$. Then $M^{1}$ is a $2-$ sphere, which may be oriented compatibly with $C P^{1}$, and $\gamma_{1}$ is the Hopf line bundle $\zeta(1)$. The cohomology ring $E^{*}\left(S_{+}^{2}\right)$ is isomorphic to $E_{*}[v] /\left(v^{2}\right)$, where $v=v^{E}(\zeta(1))=s_{2}^{E}$, and $S^{2}$ is 2 -generated with 2-rank 1 . Of course the second bundle $\gamma\left(a_{1}\right)$ is isomorphic to $\zeta(1)^{a(1,2)}$ for some 1-term sequence $a_{1}=(a(1,2))$. 
The construction is now complete, and the $k$ th stage depends only on a list $\left(a_{1}, \ldots, a_{k-1}\right)$. It is occasionally helpful to interpret the first bundle $\mathbb{C}$ as $\gamma\left(a_{0}\right)$.

We refer to the sequence $\left(M^{k}: k \leq n\right)$ of oriented manifolds as a Bott tower of height $n$ (which may be infinite); it is determined by the list $a=\left(a_{1}, \ldots, a_{n-1}\right)$, containing $n(n-1) / 2$ integers. If we choose the projective form of $M^{k}$ for every $k$, we obtain a tower of nonsingular algebraic varieties, whose orientations coincide with those described above. Following Section 7, every Bott tower involves projections $p_{k}: M^{k} \rightarrow M^{k-1}$, sections $r_{k}$ and $\widetilde{r}_{k}: M^{k-1} \rightarrow M^{k}$, and quotient maps $q_{k}: M^{k} \rightarrow T\left(a_{k-1}\right)$, for each $1 \leq k \leq n$.

Proposition 3.1. For any $1 \leq k \leq n$ there is a diffeomorphism $\phi_{k}: Q_{k} \rightarrow M^{k}$, which pulls $\gamma_{j}$ back to $\mu_{j}$ for each $1 \leq j \leq k$.

Proof. We proceed by induction on $k$, noting that $\phi_{1}$ is defined by factoring out the action of $T^{1}(a)=T$ on the domain of the canonical projection $S^{3} \rightarrow C P^{1}$. So $\phi_{1}^{*} \gamma_{1}=\mu_{1}$ by definition.

For $k \geq 1$ we assume that $\phi_{k}$ has been constructed with the stated properties. Then $\phi_{k}^{*} \gamma\left(a_{k}\right)$ is given by

$$
\left(S^{3}\right)^{k} \times_{T^{k}(a)} \mathbb{C} \longrightarrow Q_{k},
$$

where $T^{k}(a)$ acts on $\mathbb{C}$ by $w \mapsto u_{1}^{-a(1, k+1)} \ldots u_{k}^{-a(k, k+1)} w$. It follows that the projectivisation $C P\left(\phi_{k}^{*}\left(\mathbb{C} \oplus \gamma\left(a_{k}\right)\right)\right)$ coincides with $Q_{k+1}$, and we define $\phi_{k+1}$ to be the resultant bundle map to $C P\left(\mathbb{C} \oplus \gamma\left(a_{k}\right)\right)$. Then $\phi_{k+1}^{*} \gamma_{j}$ takes the the required form for $1 \leq j \leq k+1$.

Bearing Proposition 3.1 in mind, we shall treat $M^{k}$ and $Q_{k}$ as interchangeable, and relate their properties by Proposition 3.1 as necessary. For example, the projections $p_{k}$ and the sections $r_{k}$ and $\widetilde{r}_{k}$ correspond to special cases of (2.9), in which $l=1$ and $h(1)=k$. Also, the complex structure on the projective form of $M^{k}$ coincides with that of (2.15). Furthermore, the cohomology ring of $M^{k}$ may be computed as follows, in agreement with Corollary 2.10.

Proposition 3.2. For any complex oriented ring spectrum $E$, the $E_{*}$-algebra $E^{*}\left(M_{+}^{k}\right)$ is isomorphic to $E_{*}\left[v_{1}^{E}, \ldots, v_{k}^{E}\right] / I_{k}^{E}$, where $I_{k}^{E}$ denotes the ideal

$$
\left(\left(v_{j}^{E}\right)^{2}-v^{E}(\gamma) v_{j}^{E}: 1 \leq j \leq k\right) ;
$$

in particular, $E^{2 r}\left(M_{+}^{k}\right)$ is the free $E_{*}$-module generated by monomials $v_{R}^{E}$, as $R \subseteq[k]$ ranges over the subsets of cardinality $r$, and $E^{*}\left(M_{+}^{k}\right)$ has total rank $2^{r}$.

Proof. The multiplicative structure follows from $k-1$ applications of Lemma 7.2 ; the resulting relations imply the additive structure immediately.

In the crucial cases $E=H$ and $K$, we follow Section 7 by writing $v_{j}^{E}$ as $x_{j}$ in $H^{2}\left(M_{+}^{k} ; \mathbb{Z}\right)$ and $g_{j}$ in $K^{2}\left(M_{+}^{k}\right)$ respectively, for $1 \leq j \leq k$. The ideals $I_{k}^{H}$ and $I_{k}^{K}$ are then described explicitly by (7.8). The structure of $H^{*}\left(M_{+}^{k} ; \mathbb{Z}\right)$ shows that the Euler characteristic of $M^{k}$ is $2^{k}$, and is independent of $a$; this may also be confirmed by straightforward geometric argument.

By way of example we consider the tower $\left(B_{k}: 0 \leq k\right)$, whose list satisfies $a_{k}=(0, \ldots, 0,1)$ for all $k \geq 1$. We studied this case in [19], where we explained 
its significance for complex cobordism theory. In later work [5] we interpreted the points of $B_{k}$ as complete flags $0<U_{1}<\cdots<U_{k}<\mathbb{C}^{k+1}$, bounded below by the standard flag in the sense that the first $j$ standard basis vectors lie in $U_{j+1}$, for each $1 \leq j \leq k-1$. The resulting description of $B_{k}$ as a bounded flag manifold corresponds to the projective form $C P\left(\mathbb{C} \oplus \gamma_{k-1}\right)$, and allows us to display $B_{k}$ as a toric variety.

From this point onwards we prefer the Thom complex interpretation of Proposition 3.2, because of its relevance to our decomposition of $\Sigma M^{k}$.

Proposition 3.3. Given any Bott tower $\left(M^{k}: k \leq n\right)$, there is a homotopy equivalence

$$
h_{k}: \Sigma M^{k} \longrightarrow \Sigma S^{2} \vee \Sigma T\left(a_{1}\right) \vee \cdots \vee \Sigma T\left(a_{k-1}\right),
$$

for each $1 \leq k \leq n$.

Proof. It suffices to apply Proposition $7.10 k-1$ times; $S^{2}$ appears as $T\left(a_{0}\right)$.

With respect to Proposition 3.2, the homotopy equivalence $h_{k}$ induces the additive splitting

$$
E^{*}\left(M^{k}\right) \cong\left\langle v_{\leq 1}^{E}\right\rangle \oplus \cdots \oplus\left\langle v_{\leq k}^{E}\right\rangle
$$

where $\left\langle v_{\leq j}^{E}\right\rangle$ denotes the free $E_{*}$-submodule generated by those monomials $v_{R}^{E}$ for which $R \subseteq[j]$ and $j \in R$. By construction, $\left\langle v_{\leq j}^{E}\right\rangle$ is the image of $E^{*}\left(T\left(a_{j-1}\right)\right)$ under the injection $p_{k}^{*} \cdots p_{j+1}^{*} q_{j}^{*}$, for each $1 \leq j \leq k$; it is split by $l_{j-1}^{*} r_{j}^{*} \cdots r_{k}^{*}$, where $l_{j-1}^{*}$ is induced by the map $\Sigma T\left(a_{j-1}\right) \rightarrow \Sigma M^{j-1}$ which collapses the standard copy of $M^{j-1}$ in $T\left(\mathbb{R} \oplus \gamma\left(a_{j-1}\right)\right)$.

It is worth commenting on aspects of the case $k=2$, which is influenced by the fact that the isomorphism class of the $S O(3)$-bundle $\mathbb{R} \oplus \zeta(1)^{a(1,2)}$ depends only on the parity of $a(1,2)$. So there are diffeomorphisms $M^{2} \rightarrow S^{2} \times S^{2}$ when $a(1,2)=2 b$ is even, and $M^{2} \rightarrow S(\mathbb{R} \oplus \zeta(1))$ when $a(1,2)=2 b+1$ is odd. In $E$-cohomology, they induce isomorphisms

$$
\begin{aligned}
E_{*}\left[v_{1}, v_{2}\right] /\left(v_{1}^{2}, v_{2}^{2}-2 b v_{1} v_{2}\right) & \cong E_{*}\left[w_{1}, w_{2}\right] /\left(w_{1}^{2}, w_{2}^{2}\right) \text { and } \\
E_{*}\left[v_{1}, v_{2}\right] /\left(v_{1}^{2}, v_{2}^{2}-(2 b+1) v_{1} v_{2}\right) & \cong E_{*}\left[w_{1}, w_{2}\right] /\left(w_{1}^{2}, w_{2}^{2}-w_{1} w_{2}\right),
\end{aligned}
$$

(omitting the superscripts $E$ ), which are determined by the $2 \times 2$ matrices of their actions on the column vector $\left(v_{1}, v_{2}\right)$. Such matrices are exemplified by $\left(\begin{array}{ll}1 & 0 \\ b & 1\end{array}\right)$, for any integer $b$.

Having defined $M^{k}$ as an iterated sphere bundle, we now apply Szczarba's results [22] to its tangent bundle. We obtain an isomorphism

$$
\tau\left(M^{k}\right) \oplus \mathbb{R} \cong \mathbb{R} \oplus \bigoplus_{j=1}^{k} \gamma\left(a_{j-1}\right)
$$

of real bundles, which determines a stably almost complex structure $\tau^{\prime}$ on $M^{k}$. Since (3.5) extends over the 3 -disk bundle of $\mathbb{R} \oplus \gamma\left(a_{k-1}\right)$, this structure bounds. It therefore differs from that of (2.12) and (2.15).

Given a Bott tower of height $n$, we turn our attention to the projection $p_{n, k}: M^{n} \rightarrow M^{k}$, defined as the composition $p_{k+1} \cdots p_{n}$ for some $k \geq 1$. This is also a smooth bundle, whose fibre we wish to identify. In terms of the $Q_{k}$, we 
are considering special cases of (2.9); for the projection, we take $h(i)=k+i$ for $1 \leq i \leq n-k$, and for the inclusion of the fibre, we take $h(i)=i$ for $1 \leq i \leq k$.

Proposition 3.6. The fibre of $p_{n, k}$ is the $(n-k)$ th stage of a Bott tower $\left(\left(M^{\prime}\right)^{j}: j \leq n-k\right)$; it is determined by the list $\left(a_{1}^{\prime}, \ldots, a_{n-k-1}^{\prime}\right)$, where $a_{j}^{\prime}$ is formed from $a_{j+k}$ by deleting the first $k$ entries, for each $1 \leq j \leq n-k-1$.

Proof. When we restrict the bundle $\gamma\left(a_{k}\right)$ to a point $\left(M^{\prime}\right)^{0}$ in $M^{k}$, we obtain the trivial bundle $\mathbb{C}$, and $M^{k+1}$ pulls back to the fibre $S^{2}$ of $p_{k+1}$; we label this fibre $\left(M^{\prime}\right)^{1}$. We repeat the pullback procedure over $\left(M^{\prime}\right)^{1}$, and continue until we reach $M^{n-1}$. We find that $\gamma_{j}$ restricts trivially to $\left(M^{\prime}\right)^{n-k-1}$ for $1 \leq j \leq k$, and to $\gamma_{j-k}^{\prime}$ for $k<j \leq n-1$. Thus $\gamma\left(a_{n-1}\right)$ restricts to $\gamma^{\prime}\left(a_{n-k-1}^{\prime}\right)$, where $a_{n-k-1}^{\prime}=(a(k+1, n), \ldots, a(n-1, n))$, and $M^{n}$ pulls back to $S\left(\mathbb{R} \oplus \gamma^{\prime}\left(a_{n-k-1}^{\prime}\right)\right)$, which we label $\left(M^{\prime}\right)^{n-k}$. The construction ensures that $\left(M^{\prime}\right)^{n-k}$ is the inverse image of $\left(M^{\prime}\right)^{0}$ under $p_{n, k}$, and is therefore the required fibre.

Corollary 3.7. For each $1<k<n$, there is a commutative ladder of cofibre sequences

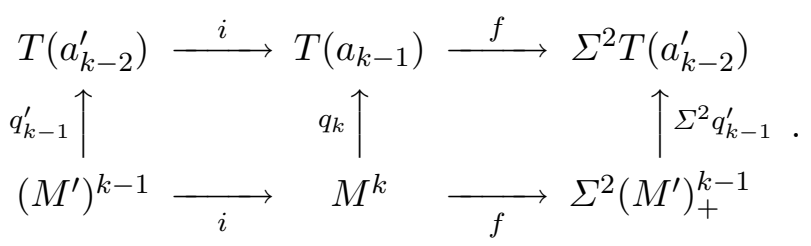

In E-cohomology, the homomorphisms induced by the upper sequence satisfy $i^{*} t_{k}^{E}=\left(t^{\prime}\right)_{k-1}^{E}$, and $f^{*}\left(\Sigma^{2} i^{*} w\left(t^{\prime}\right)_{k-1}^{E}\right)=v_{1}^{E} w t_{k}^{E}$ for every $w \in E^{*}\left(M^{k-1}\right)$. In the lower sequence they satisfy $i^{*} v_{j}^{E}=\left(v^{\prime}\right)_{j-1}^{E}$ for each $2 \leq j \leq k$, with $i^{*} v_{1}^{E}=0$, and $f^{*}\left(\Sigma^{2} i^{*} v_{R}^{E}\right)=v_{1}^{E} v_{R}^{E}$ for every $R \subseteq\{2, \ldots, k\}$, with $f^{*} s_{2}^{E}=v_{1}^{E}$.

Proof. The ladder arises by combining Proposition 3.6 with Proposition 7.11, where $X$ is $M^{k-1}$ and $Y$ is $M^{k}$. Since the upper $i$ arises from a bundle map it satisfies $i^{*} t_{k}^{E}=\left(t^{\prime}\right)_{k-1}^{E}$, yielding $i^{*} v_{k}^{E}=\left(v^{\prime}\right)_{k-1}^{E}$; the corresponding result holds for $j<k$ by projection onto $M^{j}$, noting that $\left(t^{\prime}\right)_{0}^{E}=0$. Pulling $s_{2}^{E} \otimes w t_{k}^{E}$ back around (7.14) confirms that $f^{*}\left(\Sigma^{2} i^{*} w\left(t^{\prime}\right)_{k-1}^{E}\right)=v_{1}^{E} w t_{k}^{E}$ in $E^{*}\left(T\left(a_{k-1}\right)\right.$, and applying (7.5) leads to the formula for $f^{*}$ on $E^{*}\left(\Sigma^{2}\left(M^{\prime}\right)_{+}^{k-1}\right)$.

Since all the spaces on view in Corollary 3.7 are 2-generated, the horizontal cofibre sequences are cohomologically split. The formulae for $i^{*}$ and $f^{*}$ show that the splitting of $E^{*}\left(M_{+}^{k}\right)$ takes the form

$$
\begin{aligned}
E_{*} & {\left[v_{1}^{E}, \ldots, v_{k}^{E}\right] / I_{k}^{E} \cong } \\
& \left.E_{*}\left[\left(v^{\prime}\right)_{1}^{E}, \ldots,\left(v^{\prime}\right)_{k-1}^{E}\right] /\left(I^{\prime}\right)_{k-1}^{E}\right) \oplus v_{1}^{E} E_{*}\left[\left(v^{\prime}\right)_{1}^{E}, \ldots,\left(v^{\prime}\right)_{k-1}^{E}\right] /\left(I^{\prime}\right)_{k-1}^{E},
\end{aligned}
$$

and subsumes the splitting of $E^{*}\left(T\left(a_{k-1}\right)\right)$ as

$$
\left\langle v_{\leq k}^{E}\right\rangle \cong\left\langle\left(v^{\prime}\right)_{\leq k-1}^{E}\right\rangle \oplus v_{1}^{E}\left\langle\left(v^{\prime}\right)_{\leq k-1}^{E}\right\rangle .
$$




\section{4. $K O$-Theory of Stages 1 and 2}

The $K O$-theory of toric manifolds is considerably more subtle than its complex counterpart, and is rarely free over the coefficients. Bahri and Bendersky [2] have obtained interesting results using the Adams Spectral Sequence, although their calculations are mainly additive and make little reference to the geometry of vector bundles. Our goal is to describe $K O^{*}\left(M^{k}\right)$ as a $K O_{*}$-algebra for several families of Bott towers, in terms of the bundles that we have introduced above. We also wish to understand the complexification homomorphism, for application to stably complex structures and cobordism classes in Section 6 . Here we focus on $M^{1}$ and $M^{2}$, which act as base cases for inductive calculation and are useful for establishing notation.

We appeal repeatedly to Bott's exact sequence

$$
\ldots \longrightarrow K O^{*-1}(X) \stackrel{\cdot e}{\longrightarrow} K O^{*-2}(X) \stackrel{\chi}{\longrightarrow} K^{*}(X) \stackrel{r}{\longrightarrow} K O^{*}(X) \longrightarrow \ldots,
$$

which links real and complex $K$-theory through the realification homomorphism $r$. Here, $e e$ denotes multiplication by $e$, and $\chi$ is defined by composing complexification $c$ with multiplication by $z^{-1}$. For any element $g$ of $K^{*}(X)$, the difference $g-\bar{g}$ lies in the kernel of $r$, and hence in the image of $\chi$. Moreover,

$$
c(r(g))=g+\bar{g}, \quad \text { and } \quad \chi(r(z g))=g-\bar{g} .
$$

On the other hand, $r\left(c(h)=2 h\right.$ for any $h$ in $K O^{*}(X)$. It is important to remember that $c$ is multiplicative, whereas $r$ is not.

As in Fujii [12], we define elements $u_{i}$ in $K O^{-2 i}\left(C P^{n}\right)$ by $u_{i}=r\left(z^{i+1} u(n)\right)$ for any integer $i$, where $u(n) \in K^{2}\left(C P^{n}\right)$ arises in (1.1); as a ring, $K O^{*}\left(C P^{n}\right)$ may then be described in terms of the $u_{i}$. When $n=2$, Fujii's computations stretch to an isomorphism

$$
K O^{*}\left(C P_{+}^{2}\right) \cong K O_{*}\left[u_{i}: i \in \mathbb{Z}\right] / F^{2}
$$

of $K O_{*}$-algebras, where $F^{2}$ is the ideal

$$
\left(e u_{i}, x u_{i}-2 u_{i+2}, u_{i} u_{2 j}, u_{2 i+1} u_{2 j-1}-4 u_{2(i+j)}: \text { all } i, j\right) .
$$

The relations show that $K O^{*}\left(C P^{2}\right)$ is free of additive torsion, and that $y u_{i}=$ $u_{i+4}$ for all $i$; it therefore suffices to use $u_{0}, u_{1}, u_{2}$, and $u_{3}$, as in [12], but we retain the other $u_{i}$ for notational convenience. We note that (4.3) actually defines a free $K_{*}$-module on a single generator $u_{i}$, where $z u_{i}$ is given by $u_{i+1}$ for any $i$. This is equivalent to Wood's well-known result [25] that $K O \wedge C P^{2}$ is homotopy equivalent to $K$.

Further computations lead to an isomorphism

$$
K O^{*}\left(C P_{+}^{\infty}\right) \cong K O_{*}\left[\left[u_{i}: i \in \mathbb{Z}\right]\right] / F^{\infty}
$$

of $K O_{*}$-algebras, where $F^{\infty}$ is the ideal

$$
\left(e u_{i}, x u_{i}-2 u_{i+2}, u_{i} u_{j}-u_{i-2} u_{j+2}, u_{2 i+1} u_{2 j-1}-\left(u_{0}+4\right) u_{2(i+j)}: \text { all } i, j\right) \text {. }
$$

So $K O^{2 n}\left(C P^{\infty}\right)$ is torsion-free, and isomorphic to $u_{-n} \mathbb{Z}\left[\left[u_{0}\right]\right]$ for any integer $n$, whereas $K O^{2 n+1}\left(C P^{\infty}\right)$ is zero. For any complex line bundle $\gamma$ over a 2generated complex $X$, it is convenient to interpret the pull-back of $u_{i}$ along the classifying map of $\gamma$ as a characteristic class $u_{i}(\gamma)$ in $K O^{-2 i}(X)$. 
It follows from (4.4) that $K O_{*}\left(C P^{\infty}\right)$ is torsion free, and that $K O \wedge C P^{\infty}$ is homotopy equivalent to the wedge $K O \wedge\left(\bigvee_{k \geq 0} \Sigma^{4 k} C P^{2}\right)$. This equivalence may also be deduced from the fact that a vector bundle is $K O$-orientable precisely when it is Spin [1].

We consider $\zeta^{2}$ over $C P^{\infty}$, which is universal for complex line bundles with Spin-structure, and utilise the Thom class $t^{K}$ of Lemma 7.2 in $K^{2}\left(T\left(\zeta^{2}\right)\right)$.

Lemma 4.5. There is a unique element $t_{\square}$ in $K O^{2}\left(T\left(\zeta^{2}\right)\right)$ whose complexification is given by $c\left(t_{\square}\right)=\bar{\zeta} t^{K}$; it is a Thom class, and satisfies $t_{\square}^{2}=u_{-1} t_{\square}$ in $K O^{4}\left(T\left(\zeta^{2}\right)\right)$.

Proof. The existence of a Thom isomorphism $K O^{*-2}\left(C P_{+}^{\infty}\right) \cong K O^{*}\left(T\left(\zeta^{2}\right)\right)$ confirms that $K O^{2 n}\left(T\left(\zeta^{2}\right)\right)$ is torsion free for $n \not \equiv 3 \bmod 4$. So (4.1) reduces to a short exact sequence

$$
0 \longrightarrow K O^{2 n}\left(T\left(\zeta^{2}\right)\right) \stackrel{\chi}{\longrightarrow} K^{2 n+2}\left(T\left(\zeta^{2}\right)\right) \stackrel{r}{\longrightarrow} K O^{2 n+2}\left(T\left(\zeta^{2}\right)\right) \longrightarrow 0,
$$

for $n=1$ and 2; thus $c$ is monic, and if $t_{\square}$ exists, it is unique.

The construction of $t^{K}$ implies that $\overline{t^{K}}=\bar{\zeta}^{2} t^{K}$, so that

$$
c \cdot r\left(z^{-1} \bar{\zeta} t^{K}\right)=z^{-1}\left(\bar{\zeta} t^{K}-\zeta \overline{t^{K}}\right)=0 ;
$$

hence $r\left(z^{-1} \bar{\zeta} t^{K}\right)=0$, and $t_{\square}$ exists as required. It is a Thom class because $\bar{\zeta} t^{K}$ is a Thom class and $c$ is a map of ring spectra. Moreover, $\left(t^{K}\right)^{2}=z^{-1}\left(\zeta^{2}-1\right) t^{K}$, whence

$$
c\left(t_{\square}^{2}\right)=z^{-1}\left(1-\bar{\zeta}^{2}\right) t^{K}=z^{-1}(\zeta-\bar{\zeta}) c\left(t_{\square}\right)=c\left(u_{-1} t_{\square}\right) .
$$

Thus $t_{\square}^{2}=u_{-1} t_{\square}$ in $K O^{4}\left(T\left(\zeta^{2}\right)\right)$.

The calculation of $K O^{*}(T(a))$ depends on the parity of $a$. When $a=2 b$ is even, $\zeta(1)^{a}$ is $\operatorname{Spin}(2)$-bundle, and is the pull-back of the universal example along the map $C P^{1} \rightarrow C P^{\infty}$ of degree $b$; thus $t_{\square}$ pulls back to a Thom class $t$ in $K O^{2}(T(a))$. We recall that $K O^{*}\left(S^{n}\right)$ is a free $K O_{*}$-module on the single generator $s_{n}^{K O}=s_{n} \in K O^{n}\left(S^{n}\right)$, such that $s_{n}^{2}=0$ for each $n \geq 0$.

Proposition 4.6. When a is even, $K O^{*}(T(a))$ is isomorphic to

$$
K O_{*}\left[s_{2}, t\right] /\left(s_{2}^{2}, t^{2}-a s_{2} t\right)
$$

as $K O_{*}$-algebras. When $a$ is odd, there are elements $m_{i}$ in $K O^{-2 i}(T(a))$ such that $K O^{*}(T(a))$ is isomorphic to

$$
K O_{*}\left[m_{i}: i \in \mathbb{Z}\right] / F(a, m)
$$

as $K O_{*}$-algebras, where $F(a, m)$ is the ideal

$$
\left(e m_{i}, x m_{i}-2 m_{i+2}, m_{i} m_{2 j}, m_{2 i+1} m_{2 j-1}-4 a m_{2(i+j)}: \text { all } i, j\right) .
$$

Proof. When $a$ is even, the Thom isomorphism identifies $K O^{*}(T(a))$ with the free $K O_{*}$-module on generators $t$ and $s_{2} t$. It therefore remains to evaluate $t^{2}$ in $K O^{4}(T(a))$. But $u_{-1}\left(\zeta(1)^{b}\right)=r\left(z^{-1}\left(\zeta(1)^{b}-1\right)\right)$ in $K O^{2}\left(C P^{1}\right)$, so $t^{2}=$ $\operatorname{br}\left(z^{-1}(\zeta(1)-1)\right) t=a s_{2} t$, as required.

When $a=2 b+1$ is odd, $\zeta(1)^{a}$ is no longer $K O$-orientable. We proceed by comparing the $K O$-theory of the cofibre sequences of $S^{2} \cup_{a \eta} e^{4}$ and $C P^{2}$, using the map $f(a): T(a) \rightarrow C P^{2}$ which classifies $\zeta(1)^{a}$. We define $m_{i}$ as 
$r\left(z^{i+1}\left(1-b z s_{2}^{K}\right) t^{K}\right)$ when $i$ is even, and $r\left(z^{i+1} t^{K}\right)$ when $i$ is odd. The action of $f(a)^{*}$ then yields the algebra structure, by appeal to (4.3); alternatively, we may apply complexification.

A few observations are in order. Firstly, when $a$ is even the suspension of $a \eta$ is null homotopic, so that $\Sigma T(a)$ is homotopy equivalent to $S^{3} \vee S^{5}$; equivalently, the $S O(3)$-bundle $\mathbb{R} \oplus \zeta(1)^{a}$ is trivial. Secondly, the relations of Proposition 4.6 imply that $t^{3}=0$. Thirdly, the action of $f(a)^{*}$ is computed from (4.1), and is given by

$$
f(a)^{*}\left(u_{i}\right)=\left\{\begin{array}{ll}
\left(2+b e^{2} s_{2}\right) t & i=-1 \\
b x s_{2} t & i=0 \\
x t & i=1 \\
a_{y s_{2} t} & i=2
\end{array} \text { and } f(a)^{*}\left(u_{i}\right)= \begin{cases}a m_{i} & i \equiv 0(2) \\
m_{i} & i \equiv 1(2)\end{cases}\right.
$$

for $a=2 b$ and $2 b+1$ respectively. Fourthly, when $a$ is odd, the generators $m_{i}$ may be defined more systematically as $r\left(z^{i+1} \bar{\zeta}(1)^{b} t^{K}\right)$; this description is central to Theorem 5.7 below.

Proposition 4.6 shows that $K O^{*}(T(a))$ is free over $K O_{*}$ when $a$ is even, and over $K_{*}$ when $a$ is odd. It may be interpreted in terms of spectra as providing homotopy equivalences

(4.8) $K O \wedge T(2 b) \simeq K O \wedge\left(S^{2} \vee S^{4}\right)$ and $K O \wedge T(2 b+1) \simeq K O \wedge C P^{2}$.

We may now proceed to $M^{2}$ via Proposition 3.3, which ensures that there is an additive isomorphism

$$
K O^{*}\left(M^{2}\right) \cong K O^{*}\left(S^{2}\right) \oplus K O^{*}(T(a))
$$

of $K O_{*}$-modules. It remains to describe the products in $K O^{*}\left(M^{2}\right)$. To prepare for our eventual notation, we write $p_{2}^{*} s_{2}$ as $d_{1}$ in $K O^{2}\left(M^{2}\right)$ and $q_{2}^{*} t$ as $d_{2}$ in $K O^{2}\left(M^{2}\right)$, when $a$ is even; when $a$ is odd, we write $q_{2}^{*} m_{i}$ as $n_{i}$ in $K O^{-2 i}\left(M^{2}\right)$, for all $i$.

Proposition 4.10. When a is even, $K O^{*}\left(M_{+}^{2}\right)$ is isomorphic to

$$
K O_{*}\left[d_{1}, d_{2}\right] /\left(d_{1}^{2}, d_{2}^{2}-a d_{1} d_{2}\right)
$$

as $K O_{*}$-algebras; when a is odd, it is isomorphic to

$$
K O_{*}\left[d_{1}, n_{i}: i \in \mathbb{Z}\right] /\left(F(a, n), d_{1}^{2}, d_{1} n_{2 i}, d_{1} n_{2 i+1}-2 n_{2 i}\right) .
$$

Proof. It suffices to combine Proposition 4.6 with (4.9). When $a$ is odd, the extra relations follow by applying complexification, and noting that $n_{i}$ restricts to 0 on $M^{1}$ for all $i$.

The odd case is crucial for Theorem 5.7, where it acts as base case (and motivating example) for the inductive proof. The following corollary helps us to enumerate stably complex structures on $M^{2}$ in Section 6 , and is immediate.

Corollary 4.11. In both cases, $K O^{-2}\left(M^{2}\right)$ is isomorphic to $\mathbb{Z}^{2}$ as abelian groups; bases are given by $\left\{x d_{1}, x d_{2}\right\}$ when a is even, and $\left\{x d_{1}, n_{1}\right\}$ when $a$ is odd. 
The isomorphisms of (3.4) extend to $K O^{*}\left(M^{2}\right)$, and may be described in terms of (4.8) and Proposition 4.10.

\section{KO-Theory of Bott Towers}

We now return to the Bott tower $\left(M^{k}: k \leq n\right)$, determined by the list $a=\left(a_{1}, \ldots, a_{n-1}\right)$, and study inductive procedures for computing the $K O_{*^{-}}$ algebra structure of $K O^{*}\left(M^{k}\right)$ in favourable cases.

The work of Bahri and Bendersky [2] identifies the effect of smashing $M^{k}$ with the spectrum $K O$, and leads to a homotopy equivalence

$$
K O \wedge N_{+}^{2 n} \simeq K O \wedge \bigvee_{p, q=0}^{n, n-2}\left(\bigvee^{\alpha_{p}} S^{2 p} \vee \bigvee^{\beta_{q}} \Sigma^{2 q} C P^{2}\right)
$$

for any toric manifold $N^{2 n}$. The BB-numbers $\alpha_{p}$ and $\beta_{q}$ enumerate the summands for each $p$ and $q$ respectively. Bahri and Bendersky prove that their numbers are determined by the structure of $H^{*}\left(N^{2 n} ; \mathbb{F}_{2}\right)$ over $\mathcal{A}(1)$, the subalgebra of the Steenrod algebra generated by $S q^{1}$ and $S q^{2}$. Two types of $\mathcal{A}(1)$-module are involved; the first is $\Sigma^{2 p} \mathcal{M}_{1}$, with one $2 p$-dimensional generator on which $S q^{1}$ and $S q^{2}$ act trivially, and the second is $\Sigma^{2 q} \mathcal{M}_{2}$, with one $2 q$-dimensional generator $x$ such that $S q^{1} x=0$ and $S q^{2} x \neq 0$. Then $H^{*}\left(N^{2 n} ; \mathbb{F}_{2}\right)$ decomposes as a direct sum of these two types; the number of summands $\Sigma^{2 p} \mathcal{M}_{1}$ is $\alpha_{p}$, and the number of summands $\Sigma^{2 q} \mathcal{M}_{2}$ is $\beta_{q}$.

The additive part of our calculations recover (5.1) for two particular families of Bott towers, and provide representative bundles for the generators of $K O^{*}\left(M^{k}\right)$ as a geometrical bonus. We also point out how the BB-numbers depend on the parity of the entries in $a$. Our families actually illustrate the extreme cases, which range from $\beta_{q}=0$ for all $q$, to $\alpha_{p}=0$ for all $p>1$.

We begin by reverting to the notation of Section 7 , and consider the complex line bundle $\gamma=\gamma_{1}^{a(1)} \otimes \cdots \otimes \gamma_{m}^{a(m)}$ over the 2-generated complex $X$.

When $a(j)=2 b(j)$ is even for all $1 \leq j \leq m$, we write $\gamma_{1}^{b(1)} \otimes \cdots \otimes \gamma_{m}^{b(m)}$ as $\gamma^{1 / 2}$. So $\gamma$ is $\operatorname{Spin}(2)$, and is obtained by pulling the universal example of Lemma 4.5 back along the classifying map for $\gamma^{1 / 2}$. In particular, we obtain a Thom class $t \in K O^{2}(T(\gamma))$; it satisfies $t^{2}=u_{-1}\left(\gamma^{1 / 2}\right) t$, where $u_{-1}\left(\gamma^{1 / 2}\right)=r\left(z^{-1}\left(\gamma^{1 / 2}-1\right)\right)$ in $K O^{2}(X)$, and

$$
c(t)=\prod_{j \leq m}\left(1+z g_{j}\right)^{-b(j)} t^{K}
$$

in $K^{0}(T(\gamma))$. We may now generalise the even case of Proposition 4.10.

Proposition 5.2. The $K O_{*}$-algebra $K O^{*}\left(Y_{+}\right)$is a free module over $K O^{*}\left(X_{+}\right)$ on generators 1 and $d_{m+1}$, which have dimensions 0 and 2 respectively; the multiplicative structure is determined by the single relation

$$
d_{m+1}^{2}=r\left(z^{-1}\left(\prod_{j \leq m}\left(1+z g_{j}\right)^{b(j)}-1\right)\right) d_{m+1},
$$

and $d_{m+1}$ restricts to a generator on the fibre $S^{2} \subset Y$. 
Proof. We repeat the arguments of Lemma 7.2(2) with $q^{*} t=d_{m+1}$ in $K O^{2}(Y)$, and apply the remarks above.

It is sometimes preferable to leave (5.3) in the form $d_{m+1}^{2}=u_{-1}\left(\gamma^{1 / 2}\right) d_{m+1}$, and aim to express $u_{-1}\left(\gamma^{1 / 2}\right)$ as a polynomial in the elements $r\left(z^{i} g_{j}\right)$. This does not follow automatically from (5.3), because $r$ is not multiplicative. The simplest example is $X=S^{2}$, where $\gamma^{1 / 2}$ is given by $\zeta^{b(1)}$ and $u_{-1}\left(\zeta^{b(1)}\right)$ reduces to $2 b(1) s_{2}$ in $K O^{2}\left(S^{2}\right)$. We then recover the first part of Proposition 4.10.

If one or more of the integers $a(j)$ is odd, the situation is less amenable. For our current purposes, it is enough to recall that $T(\gamma)$ admits a canonical complex line bundle $\lambda$, defined by $v^{H}(\lambda)=t^{H}$. So $t^{K}$ is represented by $z^{-1}(\lambda-1)$ in $K^{2}(T \gamma)$. The classes $u_{i}(\lambda)$ in $K O^{-2 i}(T(\gamma))$ play a major rôle in describing $K O^{*}\left(Y_{+}\right)$.

Our main structure theorems refer to two particular families of Bott towers. They are the totally even towers, for which the integers $a(i, j)=2 b(i, j)$ are even for all values of $1 \leq i<j \leq n$, and the terminally odd towers, for which the integers $a(j-1, j)=2 c(j)+1$ are odd for every $1 \leq j \leq n$. It is possible to deal with other cases by combining the two approaches.

Theorem 5.4. For any totally even Bott tower $\left(M^{k}: k \leq n\right)$, the $K O_{*}$-algebra $K O^{*}\left(M_{+}^{k}\right)$ is isomorphic to $K O_{*}\left[d_{1}, \ldots, d_{k}\right] / J_{k}^{t e}$, where $J_{k}^{t e}$ denotes the ideal

$$
\left(d_{j}^{2}-r\left(z^{-1}\left(\prod_{i<j}\left(1+z g_{i}\right)^{b(i, j)}-1\right)\right) d_{j}: 1 \leq j \leq k\right)
$$

for each $1 \leq k \leq n$, the homotopy equivalence $h_{k}$ induces the $K O_{*}$-module isomorphism

$$
K O^{*}\left(M^{k}\right) \cong\left\langle d_{\leq 1}\right\rangle \oplus \cdots \oplus\left\langle d_{\leq k}\right\rangle,
$$

where $\left\langle d_{\leq j}\right\rangle$ denotes the free submodule generated by those monomials $d_{R}$ for which $R \subseteq[j]$ and $j \in R$.

Proof. In this case the proof of Theorem 3.2 adapts directly, since all the relevant $K O_{*}$-modules are free.

As before, it may be preferable to rewrite the relations of $J_{k}^{t e}$ as

$$
d_{j}^{2}=u_{-1}\left(\gamma_{1}^{b(1, j)} \otimes \cdots \otimes \gamma_{j-1}^{b(j-1, j)}\right) d_{j},
$$

and calculate $u_{-1}\left(\gamma_{1}^{b(1, j)} \otimes \cdots \otimes \gamma_{j-1}^{b(j-1, j)}\right)$ as a polynomial in $d_{1}, \ldots, d_{j-1}$ for each $1 \leq j \leq k$. Amongst other formulae in $K O^{*}\left(M^{k}\right)$, this approach yields

$$
d_{j}^{j+1}=0 \quad \text { and } \quad d_{j}^{2}=\left(a(1, j) d_{1}+\cdots+a(j-1, j) d_{j-1}\right) d_{j} \text { modulo } P_{*},
$$

where $P_{*}$ denotes the ideal generated by triple products.

Calculations for terminally odd towers are more intricate, and we begin with the additive structure. It is convenient to index the generators by finite sets $R$ of positive integers. For every such $R$, we construct $R^{+}$by adding 1 to each element, and $1 ; R^{+}$by adjoining the integer 1 to the result. We obtain the coproduct decomposition

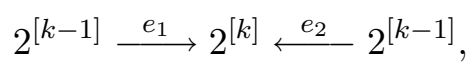


of power sets, where $e_{1}(R)=R^{+}$and $e_{2}(R)=1 ; R^{+}$. Given $R \subseteq[k-2]$ for $k \geq 2$, we construct $R ; k \subset[k]$ by adjoining the integer $k$.

So far as complex $K$-theory is concerned, we may apply this notation to the ladder (3.8). The elements $g_{R} t_{k}^{K}$ in $K^{*}\left(T\left(a_{k-1}\right)\right)$ are of two types; those for which $R$ takes the form $S^{+}$for some $S \subseteq[k-3]$, so that $i^{*}\left(g_{R} t_{k}^{K}\right)=g_{S}^{\prime}\left(t^{\prime}\right)_{k-1}^{K}$, and those for which $R$ takes the form $1 ; S^{+}$, so that $f^{*}\left(\Sigma^{2} g_{S}^{\prime}\left(t^{\prime}\right)_{k-1}^{K}\right)=g_{R} t_{k}^{K}$. The decomposition (5.6) then corresponds to the splitting (3.9). Of course, $q_{k}^{*}\left(g_{R} t_{k}^{K}\right)=g_{R ; k}$ in $K^{*}\left(M^{k}\right)$.

We may now construct the elements we need in $K O$-theory. For every integer $i$, we define

$$
m(R ; k)_{i}=r\left(z^{i+1} \bar{\gamma}_{k-1}^{b(k)} g_{R} t_{k}^{K}\right)
$$

in $K O^{2(|R|-i)}\left(T\left(a_{k-1}\right)\right)$, as $R$ ranges over subsets of [k-2], and

$$
n(R ; j)_{i}=r\left(z^{i+1} \bar{\gamma}_{j-1}^{b(j)} g_{R ; j}\right)
$$

in $K O^{2(|R|-i)}\left(M^{k}\right)$, as $R$ ranges over subsets of $[j-2]$, with $2 \leq j \leq k$. Thus $q_{k}^{*} m(R ; k)_{i}=n(R ; k)_{i}$ for every $R \subseteq[k-2]$.

Theorem 5.7. For any terminally odd Bott tower $\left(M^{k}: k \leq n\right)$, the $K O_{*-}$ module $K O^{*}\left(M_{+}^{k}\right)$ is generated by the elements

$$
\left\{d_{1}, n(R ; j)_{i}: 2 \leq j \leq k\right\},
$$

where $R$ ranges over the subsets of $[j-2]$ and $i \in \mathbb{Z}$; the submodule of relations is generated by

for all $R, j$ and $i$.

$$
\left\{e n(R ; j)_{i}, x n(R ; j)_{i}-2 n(R ; j)_{i+2}\right\}
$$

Proof. We proceed inductively, using the commutative ladder (3.8). We assume that the result holds for terminally odd towers of height $\leq n-1$, where $n \geq 2$, and consider $\left(M^{k}: k \leq n\right)$, determined by a list $\left(a_{1}, \ldots, a_{n-1}\right)$. The tower $\left(\left(M^{\prime}\right)^{k}: k \leq n-1\right)$ is determined by the list $\left(a_{1}^{\prime}, \ldots, a_{n-2}^{\prime}\right)$, where $a_{j-1}^{\prime}$ is obtained from $a_{j}$ by deleting the first element; so it is also terminally odd, and the inductive hypothesis applies.

We may therefore assume that $K O^{*}\left(T\left(a_{k-2}^{\prime}\right)\right)$ is a free abelian group, generated by the elements $m(S ; k-1)_{i}^{\prime}$ for $S \subseteq[k-3]$ and $0 \leq i \leq 3$. So $K O^{*}\left(\Sigma^{2} T\left(a_{k-2}^{\prime}\right)\right)$ is generated by their double suspensions, and both groups are zero in odd dimensions. Since $i^{*} \gamma_{j}=\gamma_{j-1}^{\prime}$ in $K O^{0}\left(\left(M^{\prime}\right)^{k-2}\right.$ for every $2 \leq j \leq k-1$, it follows from Corollary 3.7 that $i^{*} m\left(S^{+} ; k\right)_{i}=m(S ; k-1)_{i}^{\prime}$ in $K O^{0}\left(\left(M^{\prime}\right)^{k-2}\right.$, and $f^{*}\left(\Sigma^{2} m(S ; k-1)_{i}^{\prime}=m\left(1 ; S^{+} ; k\right)_{i}\right.$ in $K O^{*}\left(T\left(a_{k}\right)\right)$, for every $S \subseteq[k-3]$. Applying $K O^{*}(-)$ to the ladder yields

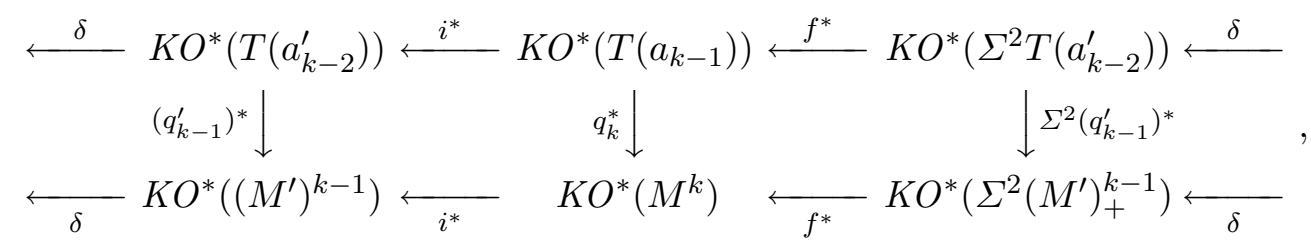

ensuring that the upper coboundary maps $\delta$ are zero for $k \geq 2$, and that the upper sequence splits as abelian groups. So $K O^{*}\left(T\left(a_{k-1}\right)\right)$ is also zero 
in odd dimensions, and generated by the $m\left(S^{+} ; k\right)_{i}$ and $m\left(1 ; S^{+} ; k\right)_{i}$ in even dimensions; but these are precisely the elements $m(R ; k)_{i}$ for $R \subseteq[k-2]$. It follows from Proposition 3.3 that $q_{k}^{*}$ injects $K O^{*}\left(T\left(a_{k-1}\right)\right)$ into $K O^{*}\left(M^{k}\right)$ as the summand generated by the elements $n(R ; k)_{i}$, for $R \subseteq[k-2]$. The abelian group structure of $K O^{*}\left(T\left(a_{k-1}\right)\right)$ ensures that complexification is monic, and therefore that

$$
e n(R ; k)_{i}=0 \quad \text { and } \quad x n(R ; k)_{i}=2 n(R ; k)_{i+2}
$$

for all $2 \leq j \leq k$. The remainder of the additive structure then follows from the inductive hypothesis. The base case $k=2$ is resolved by Proposition 4.10, with $m(\varnothing ; 2)_{i}=m_{i}$ and $n(\varnothing ; 2)_{i}=n_{i}$ for all $i$.

It follows from Theorem 5.7 that $K O^{*}\left(M^{k}\right)$ is torsion free, except for a single copy of $\mathbb{Z} / 2$ in each of the dimensions 0 and $1 \bmod 8$, generated by $e^{2} y^{i} d_{1}$ and $e y^{i} d_{1}$ respectively. This generalises the results obtained for $B_{k}$ in [9], and outlined in Example 5.13 below. Theorem 5.7 also implies that $y n(R ; j)_{i}=$ $n(R ; j)_{i+4}$ in $K O^{*}\left(M^{k}\right)$, for any $R, j$, and $i$. We may therefore restrict the choice of generators to $i=0,12$, and 3 , for example; nevertheless, we usually allow $i$ to be arbitrary for notational convenience.

In order to understand the multiplicative structure of $K O^{*}\left(M^{k}\right)$, we need to evaluate products of the generators described in Theorem 5.7.

Proposition 5.8. For any $R \subseteq[j-2]$ and $j>2$, we have that

$$
d_{1} n(R ; j)_{i}=\left\{\begin{array}{ll}
0 & \text { if } 1 \in R \\
n(1 ; R ; j)_{i} & \text { otherwise }
\end{array} ;\right.
$$

for any $R^{\prime} \subseteq\left[j^{\prime}-2\right]$, we have that

$$
\begin{aligned}
& n(R ; j)_{i} n\left(R^{\prime} ; j^{\prime}\right)_{i^{\prime}}= \\
& \quad r\left(z^{i+j+2} \bar{\gamma}_{j-1}^{b(j)} g_{R ; j}\left(\bar{\gamma}_{j^{\prime}-1}^{b\left(j^{\prime}\right)} g_{R^{\prime} ; j^{\prime}}+(-1)^{j+1} \gamma_{j^{\prime}-1}^{b\left(j^{\prime}\right)} \bar{g}_{R^{\prime} ; j^{\prime}}\right)\right) .
\end{aligned}
$$

In particular, $n(R ; j)_{i} n\left(R^{\prime} ; j\right)_{i^{\prime}}=0$ whenever $1 \in R \cap R^{\prime}$.

Proof. Theorem 5.7 implies that complexification is monic, modulo the summand $K O^{*}\left(M^{1}\right)$. Since $n(R ; j)_{i}$ restricts to 0 in $K O^{*}\left(M^{1}\right)$ for every $R, j$, and $i$, it suffices to prove the relations by applying $c$.

Now $c\left(d_{i}\right)=g_{1}$, and $c\left(n(R ; j)_{i}\right)=z^{i+1}\left(\bar{\gamma}_{j-1}^{b(j)} g_{R ; j}+(-1)^{i+1} \gamma_{j-1}^{b(j)} \bar{g}_{R ; j}\right)$ in $K^{*}\left(M^{k}\right)$. Moreover, $g_{1}^{2}=0$, so $\bar{g}_{1}=g_{1}$ and the first set of relations follows. The second set is proven similarly, by noting that

$$
c(r(x) r(y))=\operatorname{cr}(x(y+\bar{y}))
$$

for any elements $x$ and $y$ in $K O^{*}\left(M^{k}\right)$.

We would like to write (5.9) as an explicit $K O_{*}$-linear combination of the generators $d_{1}$ and $n(R ; j)_{i}$. In principle, this may be achieved by using the expressions for $\bar{g}_{m}$ and $g_{m}^{2}$ of (7.7) and (7.8) respectively; in practice, the calculations increase rapidly in complexity. Examples 5.11 and 5.13 give a more 
detailed glimpse of the difficulties which characterise the multiplicative structures described in Theorem 5.4 and Proposition 5.8. Related calculations will be presented in [11].

The following observations flow directly from Theorems 5.4 and 5.7.

Corollary 5.10. In the totally even case, the equivalence (5.1) reduces to

$$
K O \wedge M_{+}^{k} \simeq K O \wedge \bigvee_{R \subseteq[k]} S^{2|R|}
$$

thus $\alpha_{p}=\left(\begin{array}{l}k \\ p\end{array}\right)$ for all $1 \leq p \leq k$, and $\beta_{q}=0$ for all $q$. In the terminally odd case, we have

$$
K O \wedge M_{+}^{k} \simeq K O \wedge\left(S_{+}^{2} \vee \bigvee_{h=0}^{k-2} \bigvee_{R \subseteq[h]} \Sigma^{2|R|} C P^{2}\right)
$$

thus $\alpha_{p}=0$ for $2 \leq p \leq k$, and $\beta_{q}=\sum_{h=q}^{k-2}\left(\begin{array}{l}h \\ q\end{array}\right)$ for all $0 \leq q \leq k-2$.

Proof. In the totally even case, Theorem 5.4 confirms that $K O^{*}\left(M_{+}^{k}\right)$ is additively generated over $K O_{*}$ by the monomials $d_{R}=\prod_{R} g_{j}$, as $R$ ranges over the subsets of $[k]$.

In the terminally odd case, the torsion subgroup of $K O^{*}\left(M^{k}\right)$ corresponds to the summand $K O \wedge S^{2}$. The proof of Theorem 5.7 combines with (4.8) to show that

$$
K O \wedge T\left(a_{j-1}\right) \simeq K O \wedge\left(\left(S_{+}^{2}\right)^{\wedge(j-2)} \wedge C P^{2}\right)
$$

for all $1 \leq j \leq k$, where the elements $n(R ; j)_{i}$ correspond to the summand $\Sigma^{2|R|} C P^{2}$ for every $R \subseteq[j-2]$. The result now follows from Proposition 3.3 .

Corollary 5.10 illustrates the relationship between the BB-numbers and entries in the list $a$. In the totally even case, Proposition 3.2 confirms that every square is zero in $H^{*}\left(M^{k} ; \mathbb{F}_{2}\right)$, so $S q^{2}=0$; thus $\Sigma^{2 q} \mathcal{M}_{2}$ cannot occur in its decomposition, and $\beta_{q}=0$ for all $1 \leq j \leq k$, as required. In the terminally odd case, we write the mod 2 reduction of the class $x_{i}$ as $x_{i}^{\prime}$. Then Proposition 3.2 confirms that $S q^{2} x_{1}^{\prime}=0$, and $S q^{2} x_{j}^{\prime} \equiv x_{j-1}^{\prime} x_{j}^{\prime}$ modulo terms of the form $x_{i}^{\prime} x_{j}^{\prime}$ with $i \leq j-2$, for every $2 \leq j \leq k$. Thus $\alpha_{1}=1$. A simple inductive calculation reveals that $H^{2 q+2}\left(M^{k} ; \mathbb{F}_{2}\right)$ decomposes as

$$
S q^{2} H^{2 q}\left(M^{k} ; \mathbb{F}_{2}\right) \oplus H_{2 q+2},
$$

where $H_{2 q+2}$ is generated by all monomials of the form $x_{R}^{\prime} x_{j}^{\prime}$ such that $R \subseteq$ $[j-2]$ and $|R|=q$. Since $S q^{2}$ is injective on $H_{2 q+2}$, it follows that $\alpha_{p}=0$ for $2 \leq p \leq k$, and $\beta_{q}=\sum_{h=q}^{k-2}\left(\begin{array}{l}h \\ q\end{array}\right)$ for all $q$, as required.

In order to illustrate these results, we discuss two examples.

Example 5.11. Let $\left(A_{k}: k \geq 0\right)$ be the totally even tower determined by the integers $a(i, j)=0$ for $i \leq j-2$, and $a(j-1, j)=2$, for any $j \geq 1$. The relation (5.5) reduces to $d_{j}^{2}=u_{-1}\left(\gamma_{j-1}\right) d_{j}$, so we have to compute $u_{-1}\left(\gamma_{j-1}\right)$ 
in $K O^{2}\left(M^{j}\right)$; this follows inductively from an understanding of the homomorphism $f^{*}: K O^{*}\left(C P^{\infty}\right) \rightarrow K O^{*}\left(T\left(\zeta^{2}\right)\right)$, where $f$ is the map of Thom complexes classifying $\zeta^{2}$. To calculate $f^{*}$, we extend the formulae of (4.7) in case $b=1$, and find

$$
f^{*}\left(u_{i}\right)=\left\{\begin{array}{ll}
\left(2+u_{0}\right) t_{\square} & i=-1 \\
\left(e^{2}+u_{1}\right) t_{\square} & i=0 \\
\left(x+u_{2}\right) t_{\square} & i=1 \\
u_{3} t_{\square} & i=2
\end{array} .\right.
$$

We deduce that $u_{-1}\left(\gamma_{j-1}\right)$ is given by

$$
\sum_{s=0}^{\left\lfloor\frac{j-1}{4}\right\rfloor} 2 y^{s} d_{j-1} \ldots d_{j-4 s}+\sum_{s=0}^{\left\lfloor\frac{j-2}{4}\right\rfloor} e^{2} y^{s} d_{j-1} \ldots d_{j-4 s-1}+\sum_{s=0}^{\left\lfloor\frac{j-4}{4}\right\rfloor} x y^{s} d_{j-1} \ldots d_{j-4 s-3} .
$$

Example 5.13. Let $\left(B_{k}: k \geq 0\right)$ denote the terminally odd tower of bounded flag manifolds, determined by integers $a(i, j)=0$ for $i \leq j-2$ and $a(j-1, j)=1$, for all $j \geq 1$. Then each $b(j)$ is zero, and the generators $n(R ; j)_{i}$ are defined by $r\left(z^{i+1} g_{R ; j}\right)$ for every $R \subseteq[j-2]$. Products of the form $n(R ; j)_{i} \cdot n\left(R^{\prime} ; j^{\prime}\right)_{i^{\prime}}$ are given by

$$
r\left(z^{i+j+2}\left(g_{R ; j} g_{R^{\prime} ; j^{\prime}}+(-1)^{j+1} g_{R ; j} \bar{g}_{R^{\prime} ; j^{\prime}}\right)\right),
$$

and are evaluated using the formulae

$$
g_{m}^{2}=\left(\sum_{\varnothing \neq S \subseteq[m-1]} z^{|S|-1} g_{S}\right) g_{m} \quad \text { and } \quad \bar{g}_{m}=g_{m} /\left(1+z g_{m}\right)
$$

in $K^{*}\left(B_{k}\right)$, for every $1 \leq m \leq k$.

We may combine Theorems 5.4 and 5.7 to identify $K^{-2}\left(M^{k}\right)$. As explained in Section 6, these groups classify the stably almost complex structures on $M^{k}$.

Theorem 5.14. If the tower is totally even, then $\mathrm{KO}^{-2}\left(M^{k}\right)$ is isomorphic to

$$
\left(\bigoplus_{|R| \equiv 1,-1(4)} \mathbb{Z}\right) \oplus\left(\bigoplus_{|R| \equiv 0(4)} \mathbb{Z} / 2\right)
$$

where $R \subseteq[k]$; a basis is given by

$$
\left\{x y^{(|R|-1) / 4} d_{R}, y^{(|R|+1) / 4} d_{R}, e^{2} y^{|R| / 4} d_{R}\right\} .
$$

If the tower is terminally odd, then $K O^{-2}\left(M^{k}\right)$ is isomorphic to $\mathbb{Z}^{2^{k-1}}$; a basis is given by $\left\{x d_{1}, n(R ; j)_{i}\right\}$, where $R \subseteq[j-2]$ for $2 \leq j \leq k$ and $i=|R|+1$.

\section{Stably Complex Structures}

By way of conclusion, we apply our results to the study of stably complex structures on certain families of Bott towers. We consider the enumeration of those which arise from omniorientations, and discuss two particular special cases; those which restrict to almost complex structures, and those which are null-cobordant in $\Omega_{*}^{U}$. We summarise the appropriate definitions in order to establish our notation. 
Full details of the results for $\left(B_{k}: k \geq 0\right)$ in Theorems $6.3,6.6$ and 6.8 are provided in [8].

We write $B U$ and $B O$ respectively for the classifying spaces of the infinite unitary and orthogonal groups, and let $r: B U \rightarrow B S O \subset B O$ denote a specific choice of realification. The resulting maps

$$
S O / U \stackrel{f}{\longrightarrow} B U \stackrel{r}{\longrightarrow} B O
$$

induce the $K$-theory exact sequence (4.1) for connected spaces $X$. Given a smooth oriented manifold $N$, we assume that the stable tangent bundle is represented by a map $\tau^{S}: N \rightarrow B S O$, which we fix henceforth. A complex structure on $\tau^{S}$ is given by a lift $\tau$ to $B U$, and is known as a stably complex structure, or $U$-structure, on $N$; it therefore consists of a factorisation $\tau^{S}=r \cdot \tau$. We deem two $U$-structures $\tau$ and $\tau^{\prime}$ to be equivalent, or homotopic, whenever they are homotopic through lifts of $\tau^{S}$. Once $\tau$ is chosen, it leads to a complementary lift of the stable normal bundle $\nu^{S}$ of $N$, and conversely; this correspondence preserves homotopy classes.

If we begin with the opposite orientation for $N$, we obtain a second set of $U$-structures and homotopy classes. They are distinct from those described above, but correspond to them bijectively.

An almost complex structure on $N$ is given by a complex structure on the tangent bundle $\tau(N)$, and determines a compatible orientation. When $N$ is a complex manifold, it therefore admits a corresponding almost complex structure, which stabilises to the underlying $U$-structure $\tau_{\mathbb{C}}$. An arbitrary $U$-structure need not, of course, destabilise to $\tau(N)$, just as an almost complex structure need not be integrable. Henceforth, we will deal only with complex connected $N$, oriented compatibly, and will take $\tau_{\mathbb{C}}$ to be the distinguished $U$-structure. As explained in [20], we may then define a bijection between $K O^{-2}(N)$ and the homotopy classes of $U$-structures on $N$. To each $\Delta \in K O^{-2}(N)$ there corresponds a homotopy class of complex structures on the trivial bundle $\mathbb{R}^{2 L}$, for suitably large $L$, and the bijection associates the $U$-structure $\tau:=\tau_{\mathbb{C}} \oplus \mathbb{R}^{2 L}$ to $\Delta$. In other words, $\Delta\left(\tau, \tau_{\mathbb{C}}\right)$ is the difference element of $\tau$; its image under $\chi$ is represented by the virtual bundle $\tau-\tau_{\mathbb{C}}$ in $K^{0}(N)$.

So Theorem 5.14 identifies the totality of $U$-structures on the Bott tower $\left(M^{k}: k \leq n\right)$. In the terminally odd case, $\chi$ is monomorphic and the structures may be enumerated by identifying $\tau_{\mathbb{C}}$ as an element of $K^{0}\left(M^{k}\right)$, then varying $\tau-\tau_{\mathbb{C}}$ over the image of $\chi$. This strategy was applied to the tower of bounded flag manifolds $\left(B_{k}: 0 \leq k\right)$ in $[9]$.

For more general purposes, it helps to follow the lead of Section 2, and define a complex structure on an arbitrary vector bundle $\theta$ as an isomorphism $g$ from $\theta$ to a complex vector bundle $\xi$. The action of $i$ on the fibres of $\theta$ is given by conjugating its action on $\xi$ by $g$, and homotopy classes of isomorphisms correspond to homotopy classes of complex structures. An isomorphism of the form $\tau(N) \oplus \mathbb{R}^{m} \cong \xi$ therefore specifies a $U$-structure on $N$; for example, (2.15) defines the $U$-structure $\tau_{\mathbb{C}}$ underlying the projective form of $M^{k}$.

A second isomorphism $g^{\prime}: \theta \cong \xi$ defines a second complex structure $\theta^{\prime}$, which differs stably from the first by a unique difference element $\Delta\left(\theta^{\prime}, \theta\right)$ in $K O^{-2}(N)$. 
As above, its image under $\chi$ is represented by the virtual bundle $\theta^{\prime}-\theta$ in $K^{0}(N)$. Whether or not $\chi$ is monic, $\Delta\left(\theta^{\prime}, \theta\right)$ is constructed by expressing the trivial bundle $\mathbb{R}^{2 L}$ as $\theta \oplus \theta^{\perp}$ for suitably large $L$, then taking the complex structure induced by $g^{\prime}$ on $\theta$ and by the Hermitian complement of $g$ on $\theta^{\perp}$. We are particularly interested in this situation when $g^{\prime}$ is obtained from $g$ by complex conjugation; the difference element may then be described as follows.

Lemma 6.1. For any complex vector bundle $\xi$ over $N$, the difference element $\Delta(\bar{\xi}, \xi)$ is given by $r(z(\bar{\xi}-1))$ in $K^{-2}(N)$.

Proof. It is sufficient to consider the universal bundle $v$ over a complex Grassmannian of the form $U\left(W \oplus W^{\prime}\right) / U(W) \times U\left(W^{\prime}\right)$, where $W \oplus W^{\prime}$ is isomorphic to $\mathbb{C}^{L}$ for suitably large $L$. Both $\Delta(\bar{v}, v)$ and $r(z(\bar{v}-\mathbb{C}))$ may be represented by maps into $\Omega^{2} S O\left(W \oplus W^{\prime}\right)$, obtained by adjointing Bott's original periodicity maps. Details of these are in [7], as are the techniques for proving that the two maps are homotopic.

For any Bott tower $\left(M^{k}: k \leq n\right)$, we write $o(a, k)$ (or $o(k)$ when the list $a$ is understood or irrelevant) for the number of homotopy classes of $U$-structures which arise from an omniorientation on $M^{k}$. Thus $1 \leq o(k) \leq 2^{2 k}$. Applying Lemma 6.1 and the splitting (7.9) to the $U$-structure $\tau_{\mathbb{C}}$ of $(2.15)$ identifies the corresponding difference elements as

$$
\begin{gathered}
\sum_{j=1}^{k} \delta_{j} \Delta\left(\gamma_{j}, \bar{\gamma}_{j}\right)+\sum_{j=1}^{k} \epsilon_{j} \Delta\left(\left(\bar{\gamma}\left(a_{j-1}\right)-\bar{\gamma}_{j}\right),\left(\gamma\left(a_{j-1}\right)-\gamma_{j}\right)\right)= \\
\sum_{j=1}^{k}\left(\delta_{j}+\epsilon_{j}\right) r\left(z^{2} g_{j}\right)-\sum_{j=1}^{k} \epsilon_{j} r\left(z^{2} \prod_{i<j}\left(g_{i}+1\right)^{a(i, j)}\right),
\end{gathered}
$$

where $\delta_{j}$ and $\epsilon_{j}$ are 0 or 1 for all $1 \leq j \leq k$.

When $k=1$, these reduce to $0, x d_{1}$ and $2 x d_{1}$ in $K O^{-2}\left(M^{1}\right)$, so that $o(1)=3$. When $k=2$, Corollary 4.11 shows that we obtain the same elements, together with their translates by

$$
x d_{2}, \quad x\left(d_{2}-a(1,2) d_{1}\right), \quad \text { and } \quad x\left(2 d_{2}-a(1,2) d_{1}\right)
$$

when $a(1,2)$ is even, and

$$
n_{2,1}, \quad n_{2,1}-a(1,2) x d_{1}, \quad \text { and } \quad 2 n_{2,1}-a(1,2) x d_{1}
$$

when $a(1,2)$ is is odd. So $o(a, 2)=9,10,11$, and 12 , as $a(1,2)=0, \pm 1, \pm 2$, and $|a(1,2)| \geq 3$ respectively.

The calculations increase rapidly in complexity for general values of $a(i, j)$. Nevertheless, certain families of special cases yield interesting conclusions.

Theorem 6.3. For any Bott tower $\left(M^{k}: k \leq n\right)$, we have that

$$
3^{k} \leq o(k) \leq 3 \cdot 4^{k-1}
$$

for each $1 \leq k \leq n$. The maximum is attained by any tower for which the inequality $|a(k-1, k)| \geq 3$ holds for all $k$, and the minimum by the tower 
$\left(\left(C P^{1}\right)^{k}: k \geq 0\right)$; the tower of bounded flag manifolds $\left(B_{k}: k \geq 0\right)$ satisfies

$$
o(k)=\sum_{i=0}^{\lceil k / 2\rceil}\left(\begin{array}{c}
k+1 \\
2 i
\end{array}\right) 2^{k-i} .
$$

Proof. We proceed by induction on $k$, having resolved the cases $k=1$ and 2 above. We assume first that $|a(k-1, k)| \geq 3$ for all $k$, and that $o(k-1)=3 \cdot 4^{k-2}$. For $M^{k}$, the difference elements (6.2) consist of pullbacks from $M^{k-1}$, plus their translates by the three nonzero elements

$$
\left(\delta_{k}+\epsilon_{k}\right) r\left(z^{2} g_{k}\right)+\delta_{k} r\left(z^{2} \prod_{j<k}\left(g_{j}+1\right)^{a(j, k)}\right) .
$$

These map to $\left(\delta_{k}+\epsilon_{k}\right)\left(g_{k}-\bar{g}_{k}\right)+\delta_{k}\left(\prod_{j<k}\left(g_{j}+1\right)^{a(j, k)}-\prod_{j<k}\left(\bar{g}_{j}+1\right)^{a(j, k)}\right)$ under complexification, where $-\delta_{k} a(k-1, k)\left(g_{k-1}-\bar{g}_{k-1}\right)$ is the only term involving $g_{k-1}$. It follows that no such translates can result in coincident difference elements when $|a(k-1, k)| \geq 3$, and the initial induction is complete.

The tower $\left(\left(C P^{1}\right)^{k}: k \geq 0\right)$, on the other hand, has $a(i, j)=0$ for all values of $i$ and $j$, and is totally even. The translation elements (6.4) then reduce to $\left(\delta_{k}+\epsilon_{k}\right) x d_{k}$, creating one coincidence for each element pulled back from $\left(C P^{1}\right)^{k-1}$; this maximises the possible coincidences, and leads to $o(k)=$ $3 o(k-1)$. So $o(k)=3^{k}$, represented by the difference elements $\sum_{j=1}^{k} \omega_{j} x d_{j}$, where $\omega_{j}=0,1$, or 2 for each $j$.

The tower $\left(B_{k}: k \geq 0\right)$ has $a(j-1, j)=1$ for all $j<k$, and $a(i, j)=0$ otherwise. Being terminally odd, we may follow Theorem 5.14, and work with the complexifications

$$
g_{k}-\bar{g}_{k},-\left(g_{k-1}-\bar{g}_{k-1}\right)+\left(g_{k}-\bar{g}_{k}\right) \text {, and }-\left(g_{k-1}-\bar{g}_{k-1}\right)+2\left(g_{k}-\bar{g}_{k}\right),
$$

of the translation elements (6.4). These yield two coincidences for each element of the $(k-2)$ th stage. In other words, $o(k)$ satisfies the difference equation $o(k)=4 o(k-1)-2 o(k-2)$ for each $k \geq 2$. Using the initial conditions provided by $k=1$ and 2, we may then apply standard techniques [17] to deduce the required formula. The same arguments work when $a(j-1, j)=-1$ and $a(i, j)=0$ for $i \neq j-1$.

We emphasise that these results assume that an orientation has been fixed for $M^{k}$, as do Theorems 6.6 and 6.8 below.

It transpires that the $U$-structure $\tau^{\prime}$ of (3.5) is amongst those induced by an omniorientation, whose difference element satisfies $\delta_{j}=1$ and $\epsilon_{j}=0$ in (6.2), for all $1 \leq j \leq k$.

Theorem 6.5. For any Bott tower $\left(M^{k}: k \leq n\right)$, the difference element $\Delta\left(\tau^{\prime}, \tau_{\mathbb{C}}\right)$ is given by $\sum_{j=1}^{k} r\left(z^{2} g_{j}\right)$ in $K O^{-2}\left(M^{k}\right)$.

Proof. We proceed by induction on $k$, choosing $k=0$ as the base case because the elements in question are both zero.

So we assume that the result is true For $M^{k-1}$, and consider the construction of $M^{k}$. We observe that $\tau^{\prime}$ and $\tau_{\mathbb{C}}$ both arise by pulling back the corresponding $U$-structures on $M^{k-1}$, and adding the bundle of tangents along the fibres. 
By induction, the structures on $M^{k-1}$ differ by $\sum_{j=1}^{k-1} r\left(z^{2} g_{j}\right)$. Moreover, the tangents along the fibres pull back from the corresponding bundles along the fibres of the universal example over $C P^{\infty}$. In this case, $K O^{*}\left(C P^{\infty}\right)$ is torsion free, so that $\chi$ is monic and we may work in $K^{0}\left(C P^{\infty}\right)$. The relevant difference element is therefore $r\left(z^{2} u\right)$, and pulls back to $r\left(z^{2} g_{k}\right)$ over $M^{k}$. Adding the results yields the required formula.

The structure $\tau_{\mathbb{C}}$ is the stabilisation of an almost complex structure, and we would like to estimate how many others that are induced by an omniorientation share this property. We recall from Section 3 our observation that the Euler characteristic $e\left(M^{k}\right)$ is $2^{k}$.

According to Thomas [23], the structures we seek are precisely those whose $k$ th Chern class coincides with $e\left(M^{k}\right)$, and therefore with $c_{k}\left(\tau_{\mathbb{C}}\right)$. We may compute the latter by combining (7.9) with (2.15) and writing the total Chern class $c\left(\tau_{\mathbb{C}}\right)$ as

$$
\left(1-2 x_{1}\right) \prod_{j=2}^{k}\left(1+a(1, j) x_{1}+\cdots+a(j-1, j) x_{j-1}-2 x_{j}\right) .
$$

We deduce that $c_{k}\left(\tau_{\mathbb{C}}\right)=(-2)^{k} x_{1} \ldots x_{k}$. This confirms the value of $e\left(M^{k}\right)$, and shows that the orientation class defined by the complex structure on the projective form of $M^{k}$ is the dual of $(-1)^{k} x_{1} \ldots x_{k}$ in $H_{2 k}\left(M^{k}: \mathbb{Z}\right)$.

Theorem 6.6. For any Bott tower $\left(M^{k}: k \leq n\right)$, the omniorientations induce $2^{k-1}$ distinct almost complex structures on $\bar{M}^{k}$, for each $1 \leq k \leq n$.

Proof. We may build up the total Chern class of every $U$-structure on $M^{k}$ by analogy with the proof of Theorem 6.3 ; when $k=1$ we obtain $1-2 x_{1}, 1+2 x_{1}$ or 1 . Only the first of these has the required $c_{1}$, confirming the result for $k=1$.

To obtain the $k$ th stage, we multiply the $(k-1)$ th stage by one of the four possible factors

$$
\begin{aligned}
1 \pm\left(a(1, k) x_{1}+\ldots+a(k-1, k) x_{k-1}\right) \quad \text { or } \\
1 \pm\left(a(1, k) x_{1}+\cdots+a(k-1, k) x_{k-1}-2 x_{k}\right) .
\end{aligned}
$$

The only way in which the monomial $x_{1} \ldots x_{k}$ (or any of its equivalent forms such as $x_{k}^{k}$ ) can occur in the final product is by selecting one of the latter two factors at this, and every previous, stage. There are $2^{k}$ such possibilities in all, distributed equally between $\pm 2^{k} x_{1} \ldots x_{k}$.

It remains only to prove that there are no repetitions amongst the $2^{k-1}$ products with sign $(-1)^{k}$. In fact all $2^{k}$ structures have distinct $c_{1}$, as a simple computation shows.

The relevance of bounded flag manifolds to complex cobordism theory was first highlighted in [19]. Somewhat surprisingly, the most important $U$-structure from this point of view is $\tau^{\prime}$, which bounds. We would therefore like to know how many bounding $U$-structures arise from the omniorientions of $M^{k}$. We denote this number by $b(k)$, and conclude with a brief analysis of its possible values. 
Theorem 6.8. For any Bott tower $\left(M^{k}: k \leq n\right)$, we have that

$$
3^{k-1} \leq b(k) \leq 3 \cdot 4^{k-1}-2^{k}
$$

for each $1 \leq k \leq n$. The towers $\left(\left(C P^{1}\right)^{k}: k \geq 0\right)$ and $\left(B_{k}: k \geq 1\right)$ satisfy

$$
b(k)=3^{k}-2^{k} \quad \text { and } \quad b(k)=\sum_{i=0}^{\lceil k / 2\rceil}\left(\begin{array}{c}
k \\
2 i-1
\end{array}\right) 2^{k-i}
$$

respectively.

Proof. The lower bound arises from Theorem 6.3 by applying Szczarba's construction [22] to deduce that every $U$-structure on $M^{k-1}$ lifts to a bounding $U$-structure on $M^{k}$. The upper bound arises from the fact that the $k$ th Chern number $c_{k}\left[M^{k}\right]$ of every bounding $U$-structure is zero. Applying (6.7) shows that $c_{k}\left[M^{k}\right] \neq 0$ for precisely $2^{k}$ distinct $U$-structures, and the inequality $b(k) \leq 3 \cdot 4^{k-1}-2^{k}$ then follows from Theorem 6.3 .

The $3^{k}$ distinct $U$-structures on $\left(C P^{1}\right)^{k}$ arise by choosing one of the three possible structures for each factor $C P^{1}$; one bounds, the other two do not. A structure on the product bounds precisely when one or more of these $k$ choices bound, yielding $b(k)=3^{k}-2^{k}$. For $B_{k}$, we note from the proof of Theorem 6.3 that

$$
b(k)=2 o(k-1)-2 o(k-2),
$$

so $b(k)$ satisfies $b(k)=4 b(k-1)-2 b(k-2)$. But there are no bounding $U$ structures on a point, and only one on $M^{1}$; so $b(0)=0$ and $b(1)=1$. Solving the difference equation gives the required formula.

Many interesting questions remain to be answered about the rôle of Bott towers in complex cobordism theory. We hope to return to these in future.

\section{Appendix: 2-Generated Complexes}

Most of the spaces with which we work are connected CW-complexes $X$ whose integral cohomology rings $H^{*}(X ; \mathbb{Z})$ are generated by a linearly independent set of 2-dimensional elements $x_{1}, \ldots, x_{m}$. We describe such an $X$ as being 2-generated, and note that $H^{2}(X ; \mathbb{Z})$ is isomorphic to the integral lattice $\mathbb{Z}^{m}$; we refer to the elements $x_{j}$ as the 2-generators of $X$, and to $m$ as its 2 -rank. We follow combinatorial convention by abbreviating the set $\{1, \ldots, m\}$ to $[m]$, and denote the product $\prod_{R} x_{j}$ by $x_{R}$ for any subset $R \subseteq[m]$. The first Chern class $v^{H}$ defines a canonical isomorphism between the multiplicative group of complex line bundles over $X$ and $H^{2}(X ; \mathbb{Z})$, and so determines line bundles $\gamma_{j}$ such that $v^{H}\left(\gamma_{j}\right)=x_{j}$, for $1 \leq j \leq m$. In general, it assigns the $m$-tuple $(a(1), \ldots, a(m))$ to the tensor product

$$
\gamma_{1}^{a(1)} \otimes \cdots \otimes \gamma_{m}^{a(m)}
$$

By definition, $X$ lies in the category of CW-complexes whose cells are even dimensional. Various observations of Hoggar [15] therefore apply to the abelian group structure of $K O^{*}(X)$, and are relevant to parts of Sections 4 and 5 .

Given any of our complex oriented ring spectra $E$, the Chern classes $v^{E}\left(\gamma_{j}\right)=$ $v_{j}^{E}$ lie in $E^{2}(X)$ for all $1 \leq j \leq m$. The corresponding Atiyah-Hirzebruch 
spectral sequence collapses for dimensional reasons, and identifies $E^{*}(X)$ as a free $E_{*}$-module, spanned by the monomials $v_{R}^{E}$; in other words, it is generated by $v_{1}^{E}, \ldots, v_{m}^{E}$ as an $E_{*}$-algebra. An important, if atypical, example is provided by $C P^{n}$, which has a single $2-$ generator by (1.1).

The following results are well-known, and are usually obtained by applying standard methods of Borel and Hirzebruch [3]. Our interests here, however, are homotopy theoretic, and involve the stable triviality of certain cofibre sequences of 2-generated complexes and associated Thom spaces. We therefore take the opportunity to establish our notation by outlining proofs in this alternative language.

We assume that $X$ is 2 -generated, and write $\gamma$ for the line bundle (7.1). We let $Y$ denote the total space $S(\mathbb{R} \oplus \gamma)$ of the 2 -sphere bundle obtained from $\gamma$ by the addition of a trivial real line bundle, and write $p$ for the projection onto $X$. Whenever $X$ is a smooth manifold, we may assume that $Y$ is also.

Lemma 7.2. The $E_{*}$-algebra $E^{*}\left(Y_{+}\right)$is a free module over $E^{*}\left(X_{+}\right)$on generators 1 and $v_{m+1}^{E}$, which have dimensions 0 and 2 respectively; the multiplicative structure is determined by the single relation

$$
\left(v_{m+1}^{E}\right)^{2}=v^{E}(\gamma) v_{m+1}^{E},
$$

and $v_{m+1}^{E}$ restricts to $s_{2}^{E}$ on the fibre $S^{2} \subset Y$.

Proof. The sphere bundle $S(\mathbb{R} \oplus \gamma)$ admits a section $r$, given by +1 in the summand $\mathbb{R}$, and the quotient of the total space by the image of $r$ is canonically homeomorphic to the Thom complex $T(\gamma)$ [21, page 66]. In the resulting cofibre sequence

$$
X \stackrel{r}{\longrightarrow} Y \stackrel{q}{\longrightarrow} T(\gamma),
$$

the quotient map $q$ identifies the fibres $S^{2} \subset Y$ and $S^{2} \subset T(\gamma)$, and $r$ has left inverse $p$. The standard coaction of $X$ on $T(\gamma)$ interacts with the diagonal on $Y$ by the commutative square

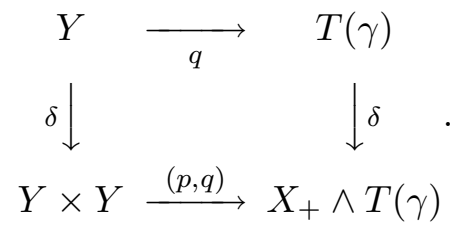

The $E$-cohomology sequence induced by (7.4) is split by $p^{*}$, and is therefore short exact. The Chern class $v^{E}$ induces a canonical Thom class $t^{E} \in E^{2}(T(\gamma))$, and so determines a Thom isomorphism $E^{*-2}\left(X_{+}\right) \cong E^{*}(T(\gamma))$, which identifies $E^{*}\left(Y_{+}\right)$as the free $E^{*}\left(X_{+}\right)$-module on generators 1 and $v_{m+1}^{E}=q^{*} t^{E}$. The diagram (7.5) confirms that products of the form $p^{*}(x) v_{m+1}^{E}$ may be written as $q^{*}\left(x t^{E}\right)$ for any $x \in E^{*}(X)$; so the action of $v_{m+1}^{E}$ is by multiplication in $E^{*}(Y)$. Since $\delta^{*}\left(v^{E}(\gamma) \otimes t^{E}\right)=\left(t^{E}\right)^{2}$, the formula for $\left(v_{m+1}^{E}\right)^{2}$ follows.

An obvious consequence of Lemma 7.2 is that $Y$ is also $2-$ generated, and has 2 -rank $m+1$. We may rewrite $v^{E}(\gamma)$ in terms of the basis $v_{1}^{E}, \ldots, v_{m}^{E}$ as

$$
v^{E}(\gamma)=F^{E}\left([a(1)]^{E}, \ldots,[a(m)]^{E}\right)
$$


in $E^{2}(X)$, using the formal group law $F^{E}$.

We shall apply these facts in the particular cases $E=H$ and $K$, denoting the elements $v_{j}^{E}$ by $x_{j}$ and $g_{j}$ respectively. Thus $z g_{j}$ is represented by the virtual bundle $\gamma_{j}-\mathbb{C}$ in $K^{0}(X)$ for $1 \leq j \leq m$, and complex conjugation acts by

$$
\bar{g}_{j}=\bar{\gamma}_{j} g_{j}=g_{j} /\left(1+z g_{j}\right)=\sum_{i=0}^{\infty}(-z)^{i} g_{j}^{i+1}
$$

the Chern character embeds $K^{*}(X)$ in the ring $H^{*}\left(X ; \mathbb{Q}\left[z, z^{-1}\right]\right)$ by $\operatorname{ch}\left(g_{j}\right)=$ $z^{-1}\left(e^{z x_{j}}-1\right)$, for $1 \leq j \leq m$. The cases $H$ and $K$ correspond to the additive and multiplicative formal group laws respectively, so the Chern classes (7.6) are given by

$$
\begin{aligned}
& v^{H}(\gamma)=a(1) x_{1}+\cdots+a(m) x_{m} \quad \text { and } \\
& v^{K}(\gamma)=z^{-1}\left(\prod_{j \leq m}\left(1+z g_{j}\right)^{a(j)}-1\right) .
\end{aligned}
$$

These are compatible under the action of the Chern character.

The universal example of Lemma 7.2 is given by $X=C P^{\infty}$ and $\gamma=\zeta$; it follows that $T(\gamma)$ is also homeomorphic to $C P^{\infty}$, and that $Y$ is homotopy equivalent to $C P^{\infty} \vee C P^{\infty}$. Then $E^{*}\left(Y_{+}\right)$is free over $E_{*}[[v]]$ on generators 1 and $v^{\prime}$, with $\left(v^{\prime}\right)^{2}=v v^{\prime}$. The general case may be deduced from this example by pulling back along the classifying map for $\gamma$. Of course, we may restrict the universal example to any skeleton $X=C P^{n}$, in which case $T(\gamma)$ is $C P^{n+1}$.

There is a second section $\widetilde{r}: X \rightarrow Y$, defined by $-1 \in \mathbb{R}$. The resulting composition $q \cdot \widetilde{r}: X \rightarrow T(\gamma)$ reduces to the inclusion of the zero-section, giving $\widetilde{r}^{*} t^{E}=v^{E}(\gamma)$.

The usual approach to Lemma 7.2 proceeds by identifying $S(\mathbb{R} \oplus \gamma)$ with its projective form $C P(\mathbb{C} \oplus \gamma)$. The corresponding canonical line bundle has first Chern class $v_{m+1}^{E}$, and is isomorphic to $\gamma_{m+1}$; it restricts to the Hopf bundle $\zeta(1)$ over the fibre $C P^{1}$. So $\gamma_{m+1}$ is a summand of the pullback $\mathbb{C} \oplus \gamma$ over $Y$, and has orthogonal complement $\bar{\gamma}_{m+1} \otimes \gamma$ with respect to the standard inner product. The associated splitting

$$
\mathbb{C} \oplus p^{*} \gamma \cong \gamma_{m+1} \oplus\left(\bar{\gamma}_{m+1} \otimes p^{*} \gamma\right)
$$

gives rise to the relation (7.3), and will be useful in Section 6 .

The cofibre sequence (7.4) also leads to the familiar relationship between the homotopy types of $X$ and $Y$.

Proposition 7.10. There is a homotopy equivalence

$$
h: \Sigma Y \longrightarrow \Sigma X \vee \Sigma T(\gamma)
$$

of suspensions.

Proof. We define $h$ as the sum $\Sigma p+\Sigma q$, and construct a homotopy inverse $\Sigma X \vee \Sigma T(\gamma) \rightarrow \Sigma Y$ by forming the wedge of $\Sigma r$ with the map $l: \Sigma T(\gamma) \rightarrow \Sigma Y$ which collapses the standard copy of $X$ in $T(\mathbb{R} \oplus \gamma)$. 
The equivalence $h$ induces an isomorphism in $E$-cohomology, which realises the module structures of Lemma 7.2 by splitting $E^{*}\left(Y_{+}\right)$as $E^{*}\left(X_{+}\right) \oplus\left(v_{m+1}^{E}\right)$. In the universal example, $h$ is a self equivalence of $\Sigma C P^{\infty} \vee \Sigma C P^{\infty}$ and desuspends.

We shall need an extension of Lemma 7.2, in the situation when $X$ itself is the total space of a bundle $\theta$ over $S^{2}$, with fibre $X^{\prime}$. We write $\gamma^{\prime}$ for the pullback of $\gamma$ to $X^{\prime}$, and $Y^{\prime}$ for the total space $S\left(\mathbb{R} \oplus \gamma^{\prime}\right)$; thus $Y^{\prime}$ is also the fibre of the projection $Y \rightarrow S^{2}$.

Proposition 7.11. With the data above, there is a homotopy commutative ladder of cofibre sequences

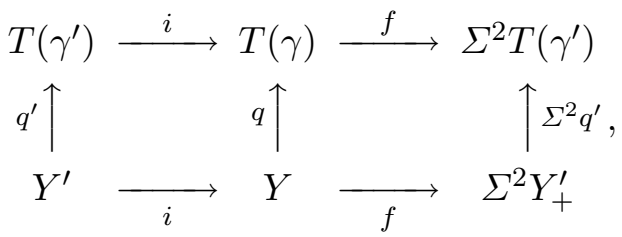

where the maps $i$ are induced by inclusion of the fibre, and the maps $f$ are quotients.

Proof. We may construct $X$ from two copies of $\mathbb{D}^{2} \times X^{\prime}$ by identifying them along their boundaries $S^{1} \times X^{\prime}$ via the characteristic function of $\theta$. Then $X / i\left(X^{\prime}\right)$ is homeomorphic to $\Sigma^{2} X_{+}^{\prime}$. The same argument applies to $Y / i\left(Y^{\prime}\right)$, yielding cofibre sequences

$$
X^{\prime} \stackrel{i}{\longrightarrow} X \stackrel{f}{\longrightarrow} \Sigma^{2} X_{+}^{\prime} \quad \text { and } \quad Y^{\prime} \stackrel{i}{\longrightarrow} Y \stackrel{f}{\longrightarrow} \Sigma^{2} Y_{+}^{\prime} .
$$

The sections $r^{\prime}: X^{\prime} \rightarrow Y^{\prime}$ and $r: X \rightarrow Y$ are compatible with the inclusions $i$, and the ladder follows by taking quotient maps $q^{\prime}$ and $q$.

The naturality of the ladder (7.12) leads to a commutative square

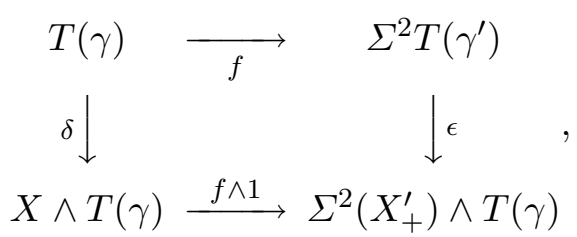

where $\epsilon$ is the Thom complexification of the bundle map obtained by pulling $\mathbb{R}^{2} \times \gamma$ back along the restricted diagonal $X^{\prime} \rightarrow X^{\prime} \times X$. Alternatively, the square may be considered as the quotient of the reduced diagonal $T(\gamma) \rightarrow X \wedge T(\gamma)$ by its restriction $T\left(\gamma^{\prime}\right) \rightarrow X^{\prime} \wedge T(\gamma)$.

The first sequence of (7.13) induces the Wang long exact sequence of $\theta$ in $E$ cohomology, for any multiplicative spectrum $E$. Standard homotopy theoretic arguments [24, pages 316-319] show that the connecting map $\Sigma^{2} X_{+}^{\prime} \rightarrow \Sigma X^{\prime}$ is induced from the characteristic map $S^{1} \times X^{\prime} \rightarrow X^{\prime}$ by suspension.

\section{REFERENCES}

[1] Michael F Atiyah, Raoul Bott, and Arnold Shapiro. Clifford modules. Topology, 3 suppl. 1:3-38, 1964.

[2] Anthony Bahri and Martin Bendersky. The KO-theory of toric manifolds. Transactions of the American Mathematical Society, 352:1191-1202, 2000. 
[3] Armand Borel and Friedrich Hirzebruch. Characteristic classes and homogeneous spaces I. American Journal of Mathematics, 80:459-538, 1958; II. American Journal of Mathematics, 81:315-382, 1959.

[4] Raoul Bott and Hans Samelson. Application of the theory of Morse to symmetric spaces. American Journal of Mathematics, 80:964-1029, 1958.

[5] Victor M Buchstaber and Nigel Ray. Flag manifolds and the Landweber-Novikov algebra. Geometry \& Topology, http://www.maths.warwick.ac.uk/gt/, 2:79-101, 1998.

[6] Victor M Buchstaber and Nigel Ray. Tangential structures on toric manifolds, and connected sums of polytopes. International Mathematics Research Notices, 4:193-219, 2001.

[7] Henri Cartan. Périodicité des Groupes d'Homotopie Stables des Groupes Classiques, d'après Bott, volume 60 of Séminaire Henri Cartan. Ecole Normale Supérieur, 1961. 2e édition, corrigée.

[8] Yusuf Civan. Stably and almost complex structures on bounded flag manifolds. Preprint, Suleyman Demirel University, Isparta, 2004.

[9] Yusuf Civan. The Topology of Families of Toric Manifolds. PhD thesis, Manchester University, 2001.

[10] Michael W Davis and Tadeusz Januszkiewicz. Convex Polytopes, Coxeter Orbifolds and Torus Actions. Duke Mathematical Journal, 62:417-451, 1991.

[11] Adrian Dobson. The KO-Theory of Thom Complexes. PhD thesis, in preparation, University of Manchester, 2005.

[12] Michikazu Fujii. $K_{O}$-groups of projective spaces. Osaka Journal of Mathematics, 4:141$149,1967$.

[13] Brayton Gray and Nigel Ray. Splitting $C P^{\infty}$ and $B \mathbf{Z} / p^{n}$ into thom spectra. Mathematical Proceedings of the Cambridge Philosophical Society, 106:263-271, 1989.

[14] Michael Grossberg and Yael Karshon. Bott towers, complete integrability, and the extended character of representations. Duke Mathematical Journal, 76:23-58, 1994.

[15] Stuart G Hoggar. On KO-theory of Grassmannians. Quarterly Journal of Mathematics Oxford, 20:447-463, 1969.

[16] Max Karoubi. K-Theory. An Introduction, volume 226 of Grundlehren der mathematischen Wissenschaften. Springer Verlag, 1978.

[17] Walter G Kelley and Allan C Peterson. Difference Equations. Academic Press, 1991.

[18] John W Milnor and James D Stasheff. Characteristic Classes, volume 76 of Annals of Mathematics Studies. Princeton University Press, 1974.

[19] Nigel Ray. On a construction in bordism theory. Proceedings of the Edinburgh Mathematical Society, 29:413-422, 1986.

[20] Nigel Ray, Robert Switzer, and Larry Taylor. G structures, G bordism and universal manifolds. Memoirs of the American Mathematical Society, 193:1-27, 1977.

[21] Robert E Stong. Notes on Cobordism Theory. Princeton University Press, 1968.

[22] R. H. Szczarba. On tangent bundles of fibre spaces and quotient spaces. American Journal of Mathematics, 86:685-697, 1964.

[23] Emery Thomas. Complex structures on real vector bundles. American Journal of Mathematics, 89:887-908, 1967.

[24] George H Whitehead. Elements of Homotopy Theory. Graduate Texts in Mathematics. Springer Verlag, 1978.

[25] Reginald M W Wood. $K$-theory and the complex projective plane. Preprint, University of Manchester, 1964.

[26] Günter M. Ziegler. Lectures on Polytopes. Graduate Texts in Mathematics 152. SpringerVerlag, 1995. 
Department of Mathematics, Faculty of Arts and Sciences, Suleyman Demirel UNIVERSITY, 32260 ISPARTA, TURKEY

E-mail address: ycivan@fef.sdu.edu.tr

Department of Mathematic, University of Manchester, Oxford Road, ManchESTER M13 9PL, ENGLAND

E-mail address: nige@ma.man.ac.uk 